\title{
Dirac fermions in strong electric field and quantum transport in graphene
}

\author{
S. P. Gavrilov, ${ }^{1,2, *}$ D. M. Gitman, ${ }^{1, \text { 由 }}$ and N. Yokomizo, ${ }^{1, \text { 团 }}$ \\ ${ }^{1}$ Institute of Physics, University of São Paulo, CP 66318, CEP 05315-970 São Paulo, SP, Brazil \\ ${ }^{2}$ Department of General and Experimental Physics, \\ Herzen State Pedagogical University of Russia, Moyka emb. 48, 191186 St. Petersburg, Russia
}

\begin{abstract}
Our previous results on the nonperturbative calculations of the mean current and of the energymomentum tensor in QED with the $T$-constant electric field are generalized to arbitrary dimensions. The renormalized mean values are found; the vacuum polarization and particle creation contributions to these mean values are isolated in the large $T$-limit, the vacuum polarization contributions being related to the one-loop effective Euler-Heisenberg Lagrangian. Peculiarities in odd dimensions are considered in detail. We adapt general results obtained in $2+1$ dimensions to the conditions which are realized in the Dirac model for graphene. We study the quantum electronic and energy transport in the graphene at low carrier density and low temperatures when quantum interference effects are important. Our description of the quantum transport in the graphene is based on the so-called generalized Furry picture in QED where the strong external field is taken into account nonperturbatively; this approach is not restricted to a semiclassical approximation for carriers and does not use any statistical assumptions inherent in the Boltzmann transport theory. In addition, we consider the evolution of the mean electromagnetic field in the graphene, taking into account the backreaction of the matter field to the applied external field. We find solutions of the corresponding Dirac-Maxwell set of equations and with their help we calculate the effective mean electromagnetic field and effective mean values of the current and the energy-momentum tensor. The nonlinear and linear $I-V$ characteristics experimentally observed in both low and high mobility graphene samples is quite well explained in the framework of the proposed approach, their peculiarities being essentially due to the carrier creation from the vacuum by the applied electric field.
\end{abstract}

PACS numbers: $11.10 . \mathrm{Kk}, 12.20 . \mathrm{Ds}, 72.80 . \mathrm{Vp}$

\section{INTRODUCTION}

\section{A. General}

It is well-known that QFT with an external background is an adequate model for studying quantum processes in the cases when a part of the quantized field is strong enough to be treated as a given and a classical one. Numerous problems in QED and QCD with superstrong electromagnetic fields, which must be treated nonperturbatively, are at present investigated in this framework, with applications to astrophysics and condensed matter physics (e.g. graphene physics) [1-4]. In these models, one often needs to know how the external background affects admitted states of the quantized fields and to what extent the external background idealization is consistent, i.e., whether the backreaction on the external background is sufficiently small. Here we consider the quantized Dirac field interacting with an external electromagnetic background. The external electromagnetic field changes the states of the Dirac field and induces transitions between them. These changes in general occur as vacuum polarization effects combined with those due to particle creation. A uniform constant magnetic field, for instance, can produce only vacuum polarization effects, whereas

\footnotetext{
* gavrilovsergeyp@yahoo.com

$\dagger$ gitman@dfn.if.usp.br

‡ yokomizo@fma.if.usp.br
}

particle creation is due to electric-like external fields.

The first calculations of vacuum polarization effects have been carried out by Heisenberg and Euler in the case of QED with constant, uniform, parallel electric $E$ and magnetic $B$ fields, with $E \ll E_{c}=m^{2} / e \simeq 10^{16} \mathrm{~V} / \mathrm{cm}$ $\left(E_{c}\right.$ is Schwinger's critical field) and arbitrary $B$, ignoring particle creation [5]. It turns out that the vacuum polarization effects (which are local in time in the uniform background) can be described by some effective Lagrangians, in particular by the Heisenberg-Euler Lagrangian, see [6]. On the other hand, the particle creation effects are global ones, as they depend on the history of the external field action and, therefore, cannot be described by any local effective Lagrangian (this does not contradict the fact that the imaginary part of the Heisenberg-Euler effective action is related to the total number of particle created, since this action is a global quantity). A non-perturbative approach (with respect to the external background) to the particle creation in the so-called $t$-electric steps (electric-like fields that are switched on and off at initial and final time instants, respectively) was elaborated in the framework of relativistic quantum mechanics in [7], and in the framework of QFT in [1]; a more complete list of publications on the particle creation can be found in 3]. A direct way to study the influence of an external electromagnetic background on the Dirac field is calculating nonperturbatively the mean energy-momentum tensor. In case of a quasiconstant electric field (the so-called $T$-constant electric 
field 1 , i.e. a uniform electric field, constant within a time interval $T$, and zero outside it) such a problem was solved in [9, 10]. The obtained result allows one to isolate, under certain conditions, vacuum polarization effects from the particle creation effects.

Until recently, problems related to particle creation from the vacuum were of a purely theoretical interest. This is related to the fact that, due to the presence of large gaps between the upper and lower branches in the spectrum of Dirac particles, particle creation effects can be observed only in huge external electric fields of the magnitude of $E_{c}$. However, recent technological advances in laser science suggest that lasers such as those planned for the Extreme Light Infrastructure project (ELI) may be able to reach the non-perturbative regime of pair production in the near future (see review [11]). Moreover, the situation has changed completely in recent years regarding applications to condensed matter physics: particle creation became an observable effect in graphene physics, an area which is currently under intense development [12, 13]. Briefly, this is explained by two facts: first, the low-energy electronic excitations in the graphene monolayer in the presence of an external electromagnetic field can be described by the Dirac model [14], namely, by a $2+1$ quantized Dirac field in such a background (that is, dispersion surfaces are the so-called Dirac cones); and, second, the gap between the upper and lower branches in the corresponding Dirac particle spectra is very small, so that the particle creation effect turns out to be dominant (under certain conditions) as a response to the external electric-like field action on the graphene. In particular, this effect is crucial for understanding the conductivity of graphene, specially in the so-called non-linear regime [4, 15 18]. The first experimental observation of non-linear current-voltage characteristics $(I-V)$ of graphene devices and its interpretation in terms of pair-creation has been recently reported [19].

It was also shown recently in [20] that linear dispersion surfaces with the Dirac points, similar to those in the graphene monolayers, are generic in the spectrum of non-relativistic $2+1$-dimensional Hamiltonians with potentials that have the symmetry of a honeycomb structure. Thus, one may expect the discovery of new materials in which the conductivity is described by a Dirac model of $(2+1)$ massless fermions. Moreover, a mass gap in the graphene band structure can be generated by several methods. One example is given by graphene nanoribbons. In this case one has a quasi-one-dimensional spatial geometry that confines the graphene electrons to a strip of large length and small width. The confinement gap depends on the strip width and on imposed boundary conditions, see [13] for a review. The spectrum of graphene can also be gapped by explicit or spontaneous sublattice symmetry breaking, see [21] and references therein. It is

${ }^{1}$ It should be noted that particle creation in the $T$-constant electric field was first considered in Ref. [8]. an important fundamental and practical problem under current research.

It should also be noted that there is a deep connection between graphene, which has two Dirac cones, and topological insulators, which are characterized by a single Dirac cone on each surface; see [13, 22] for a review and [23] for the first experimental realization of a singlevalley Dirac system in zero-gap HgTe quantum wells. In these Dirac systems, the mass gap can be generated by an appropriate selection of material parameters that depend on the quantum well geometry. In our consideration, it is assumed that the two cones of graphene are decoupled and the system behaves like two copies of a single Dirac cone. Thus, the results obtained for graphene should also be relevant for the single Dirac cone on the surface of a topological insulator. Then we expect the interface transport properties of topological insulator to be similar to those described for graphene.

In the present article, we adapt our general results on the particle creation to the cases which are realized in the Dirac model for graphene. First, our previous results in calculating the vacuum mean current and the energymomentum tensor of the Dirac field in the $T$-constant electric field [9, 10] is extended to arbitrary dimensions, especially to $(2+1)$-d. Then we consider some of immediate applications of these results to a number of problems related to the dc conductivity of graphene in the so-called superlinear (non-perturbative) regime.

We show that the application of non-perturbative methods of QED with strong field and unstable vacuum to studying the Dirac model in graphene allows one to more adequately describe quantum transport in graphene. The matter is that usually the electronic transport in graphene, even near the Dirac point, is described within the framework of traditional methods used in condensed matter physics. For example, these are methods based on WKB description of the carriers [15, 19], or numerical methods that attend one-particle quantum mechanical description of the carriers [17]. Other numerical methods exploit a Green's function formalism of nonequilibrium statistical mechanics adopted for describing states not very far from the equilibrium [19]. In some cases the Boltzmann transport theory, based on WKB approximation, describes the system evolution quite well, and sometimes it allows one to find analytic solutions. However, these cases are restricted to the ones when the large carrier densities obey the assumption that characteristic length scales of the system be larger than typical wave lengths of carriers. Moreover, the Boltzmann theory is not adapted to describe motion of massless carries created from the vacuum. The numerical methods in their present form are not adjusted for studying the time-evolution of the system that would take the electrodynamic backreaction into account, whereas the latter may be of essential importance in the present highly non-equilibrium environment caused by the pair creation. Moreover, the electric field creates carriers in pure states, whose distribution differs significantly from 
the equilibrium distribution. This is a nonstandard situation for usual transport problems in the condensed matter physics. Thus, a proper description of the quantum transport in graphene close to the Dirac point both in the ballistic case and in the presence of a disorder is still an open problem.

Our description of the quantum transport in the graphene is based on strong-field QED, it is not restricted by a semiclassical approximation of carriers and it does not use any statistical suppositions typical of the Boltzmann transport theory. Our approach is based on exact solutions of the Dirac equation, where the strong external field is taken into account non-perturbatively. A strong field approximation used for analytical calculations is related to the consistency of the Dirac model with a given external field and we show that it is well-working under certain conditions. In fact, we study the electronic and energy transport in graphene at low carrier density and low temperatures when quantum interference effects are important.

We consider the evolution of the mean electromagnetic field in the graphene, taking into account the backreaction of the quantum matter field on the applied external field. In doing this, we use consistency restrictions that describe the regime when the backreaction can be neglected. We derive restrictions from above on the allowed strength of the external electric field and on its duration, admitted at its given strength. In making some experimental conclusions, we try to compare these restrictions with typical experimental scales. We present a generalization of the QED model with the $T$-constant external field to take into account the backreaction of the mean current to the applied electric field. We find a self-consistent solution of the Dirac-Maxwell set of equations for this generalized model and calculate the effective mean field and effective mean values of the current and the energy-momentum tensor. We show that the non-linear and linear $I-V$ experimentally observed in low and high mobility samples, respectively, can be explained in the framework of the presented consideration, and that such a behavior is a consequence of the fact that the conductivity in the graphene is essentially due to the pair creation from the vacuum by the applied electric field.

The article is organized as follows: In Subsection IB we give introductory overview of the basics of the QED with unstable vacuum and introduce the necessary general notation. In Section [I] we introduce the Dirac model in a $T$-constant background, we fix notation and collect previous results (mainly of [10, 24]) required for our work. Exact solutions of the Dirac equation for this background are presented, and we describe how they are related to the particle creation and to the mean values of field observables, following the general theory presented in [1]. In Section III we consider the vacuum mean values of the current density and the energy-momentum tensor in a $T$-constant background for the case of arbitrary dimensions. Namely, in Subsection $\amalg A$ we express the vac- uum mean values of the current density and the energymomentum tensor in terms of appropriate Green functions constructed by the help of the exact solutions of the Dirac equation in the external field. In Subsection IIIB peculiarities in odd dimensions are considered. In Section $\amalg$ IIC vacuum polarization and particle creation contributions to the mean values of the energy-momentum tensor are isolated in the large duration approximation, and the vacuum polarization contributions are related to the one-loop effective Euler-Heisenberg Lagrangian. In Subsection IIID we find renormalized mean values of the energy-momentum tensor using the zeta-function regularization and compute the vacuum polarization contributions to these mean values in arbitrary dimensions. In Subsection IIE we compute the particle creation contributions to the mean values of the current and of the energy-momentum tensor in arbitrary dimensions to generalize the four dimension results of [10]. Pair-creation contributions are finite, due to the finite duration of the field. In Section IV, our results are applied to graphene physics. Namely, in Subsection IVA the Dirac model of graphene is briefly described, and the mean values computed in the previous section are used to determine the mean current and energy-momentum tensor in the material. In Subsection IVB these results allow us to estimate scales for backreaction effects, using the appropriate solution of the Maxwell equations. We show that the superlinearity of $I-V$ observed experimentally in low mobility samples is essentially due to the pair creation from the vacuum by the applied electric field in the regime where the backreaction is negligible. In Subsection IVC we study a generalization of the model with the $T$-constant external field to taking into account the backreaction of the mean current to the applied electric field. We find a self-consistent solution of the Dirac-Maxwell set of equations (with unstable vacuum in fermion sector) and calculate the effective mean field and effective mean values of the current and of energy-momentum tensor. We show that linear $I-V$ experimentally observed in high mobility samples is due to the pair creation from the vacuum by the applied electric field in the regime where backreaction is important. In Section $\mathrm{V}$ (Summary) we briefly list the main new results obtained in the article and add some relevant comments.

\section{B. Dirac model with external background}

In order to study the Dirac field in the external background, we use a formulation of QED with unstable vacuum (the generalized Furry representation) developed in [1, 25], where a strong external field is treated nonperturbatively. In this work we consider the case of the so-called $T$-constant electric field, i.e., we assume that for $t<t_{\text {in }}$ and for $t>t_{\text {out }}$, the $T$-constant electric field is absent, therefore initial and final vacua are vacuum state of free $i n$ - and out- particles respectively. During the interval $t_{\text {out }}-t_{\text {in }}=T$, the Dirac field interacts with a constant 
uniform electric field. The initial and final vacua are different due to the difference of the initial and final value of the external electromagnetic field potentials. In the Heisenberg picture, there exists a set of creation and annihilation operators $a_{n}^{\dagger}(i n), a_{n}(i n)$ of $i n$-particles (electrons), and operators $b_{n}^{\dagger}(i n), b_{n}(i n)$ of $i n$-antiparticles (positrons), the corresponding $i n$-vacuum being $|0, i n\rangle$ at the same time there exists a set of creation and annihilation operators $a_{n}^{\dagger}$ (out), $a_{n}$ (out) of out-electrons and operators $b_{n}^{\dagger}$ (out), $b_{n}$ (out) of out-positrons, the corresponding out-vacuum being $\mid 0$, out $\rangle$,

$$
\begin{aligned}
& \left.\left.a_{n}(\text { in }) \mid 0, \text { in }\right\rangle=b_{n}(\text { in }) \mid 0, \text { in }\right\rangle=0, \forall n, \\
& \left.\left.a_{n}(\text { out }) \mid 0, \text { out }\right\rangle=b_{n}(\text { out }) \mid 0, \text { out }\right\rangle=0, \forall n .
\end{aligned}
$$

In both cases, by $n$ we denote complete sets of quantum numbers describing in- and out- particles. The in- and out-operators obey the canonical anticommutation relations:

$$
\begin{aligned}
& {\left[a_{n}(\text { in }), a_{n^{\prime}}^{\dagger}(\text { in })\right]_{+}=\left[a_{n}(\text { out }), a_{n^{\prime}}^{\dagger}(\text { out })\right]_{+}=\delta_{n, n^{\prime}},} \\
& {\left[b_{n}(\text { in }), b_{n^{\prime}}^{\dagger}(\text { in })\right]_{+}=\left[b_{n}(\text { out }), b_{n^{\prime}}^{\dagger}(\text { out })\right]_{+}=\delta_{n, n^{\prime}},}
\end{aligned}
$$

the remaining anticommutators being zero.

The $i n$-operators are associated with a complete orthonormal set of solutions $\left\{{ }_{\zeta} \psi_{n}(x)\right\}(\zeta=+$ for electrons and $\zeta=-$ for positrons) of the Dirac equation with $T$ constant electric field. Their asymptotics as $t<t_{\text {in }}$ can be classified as free particles and antiparticles. The outoperators are associated with a complete orthonormal out-set of solutions $\left\{{ }^{\zeta} \psi_{n}(x)\right\}$ of the Dirac equation with $T$-constant electric field. Their asymptotics as $t>t_{\text {out }}$ can be classified as free particles and antiparticles. The $i n$ - and out- operators are defined by the two representations of the quantum Dirac field $\Psi(x)$ in the Heisenberg representation

$$
\begin{aligned}
\Psi(x) & =\sum_{n}\left[a_{n}(\text { in })+\psi_{n}(x)+b_{n}^{\dagger}(\text { in })_{-} \psi_{n}(x)\right] \\
& \left.=\sum_{n}\left[a_{n}(\text { out })^{+} \psi_{n}(x)+b_{n}^{\dagger}(\text { out })\right)^{-} \psi_{n}(x)\right] .
\end{aligned}
$$

In- and out-solutions with given quantum numbers $n$ are related by a linear transformation of the form:

$$
{ }^{\zeta} \psi_{n}(x)=g\left(\left.{ }_{+}\right|^{\zeta}\right)+\psi_{n}(x)+g\left(-\left.\right|^{\zeta}\right)-\psi_{n}(x),
$$

where the $g^{\prime}$ s are some complex coefficients. Then a linear canonical transformation (Bogolyubov transformation) between in- and out- operators which follows from Eq. (11) is defined by these coefficients.

The vacuum mean current vector, energy and momentum vector of the quantum Dirac field $\Psi(x)$ at a time instant $t$ are defined as integrals over the spatial volume. Due to translational invariance of the external field under consideration, all these mean values are proportional to the space volume. Therefore, it is enough to calculate the vacuum mean values of the current density vector $\left\langle j^{\mu}(t)\right\rangle$ and of the energy-momentum tensor (EMT) $\left\langle T_{\mu \nu}(t)\right\rangle$,

$$
\begin{gathered}
\left\langle j^{\mu}(t)\right\rangle=\left\langle 0, i n\left|j^{\mu}\right| 0, i n\right\rangle, \\
\left\langle T_{\mu \nu}(t)\right\rangle=\left\langle 0, i n\left|T_{\mu \nu}\right| 0, i n\right\rangle .
\end{gathered}
$$

Here we stress the time-dependence of mean values (3), which does exist due to the time-dependence of the external field. The operators of the current density and the EMT are described in terms of the quantum Dirac field as follows 2

$$
\begin{aligned}
& j^{\mu}=\frac{q}{2}\left[\bar{\Psi}(x), \gamma^{\mu} \Psi(x)\right], \quad T_{\mu \nu}=\frac{1}{2}\left(T_{\mu \nu}^{c a n}+T_{\nu \mu}^{c a n}\right), \\
& T_{\mu \nu}^{c a n}=\frac{1}{4}\left\{\left[\bar{\Psi}(x), \gamma_{\mu} P_{\nu} \Psi(x)\right]+\left[P_{\nu}^{*} \bar{\Psi}(x), \gamma_{\mu} \Psi(x)\right]\right\}, \\
& P_{\mu}=i \partial_{\mu}-q A_{\mu}(x), \bar{\Psi}(x)=\Psi^{\dagger}(x) \gamma^{0},
\end{aligned}
$$

where $A_{\mu}(x)$ are electromagnetic potentials of the external field, $q$ is the particle charge (for an electron $q=-e$ ), and $\Psi(x)$ is the Heisenberg operator of the Dirac field that obeys the Dirac equation with the external background.

Note that the mean values (3) depend on the definition of the initial vacuum, $|0, i n\rangle$ and on the evolution of the electric field from the time $t_{i n}$ of switching it on up to the current time instant $t$, but they have nothing to do with the further history of the system. The renormalized vacuum mean values $\left\langle j^{\mu}(t)\right\rangle$ and $\left\langle T_{\mu \nu}(t)\right\rangle, t_{i n}<t<$ $t_{\text {out }}$ are sources in equations of motion for mean electromagnetic and metric fields, respectively. In particular, complete description of the backreaction is related to the calculation of these mean values for any $t$.

\section{DIRAC FIELD IN T-CONSTANT ELECTRIC BACKGROUND}

In what follows we are going to deal with the Dirac equation in $(d=D+1)$-dimensional Minkowski space with an external electromagnetic field $A_{\mu}(x)$,

$$
\left(\gamma^{\mu} P_{\mu}-m\right) \psi(x)=0 .
$$

Here $\psi(x)$ is a $2^{[d / 2]}$-component spinor (the brackets stand for 'integer part of'), $m$ is the mass, $q$ is the charge, the Greek index assumes values $\mu=0,1, \ldots, D$, and the gamma matrices satisfy the standard anti-commutation relations:

$$
\left[\gamma^{\mu}, \gamma^{\nu}\right]_{+}=2 \eta^{\mu \nu}, \quad \eta_{\mu \nu}=\operatorname{diag}(1,-1, \ldots,-1) .
$$

In what follows, we consider the $T$-constant field described by a vector potential with only one nonzero component $A_{1}(t)\left(A_{\mu}(t)=0, \mu \neq 1\right)$,

$$
A_{1}(t)=E \begin{cases}t_{\text {in }} & t \in \mathrm{I}=\left(-\infty, t_{\text {in }}\right), t_{\text {in }}=-T / 2, \\ t, & t \in \mathrm{Int}=\left[t_{\text {in }}, t_{\text {out }}\right] \\ t_{\text {out }}, & t \in \mathrm{II}=\left(t_{\text {out }}, \infty\right), t_{\text {out }}=T / 2 .\end{cases}
$$

\footnotetext{
${ }^{2}$ We use the relativistic units $\hbar=c=1$, in which the fine structure constant is $\alpha=e^{2} / c \hbar=e^{2}$.
} 
such that the electric field $\mathbf{E}(t)$ has also only one nonzero component, which is nonzero for $t \in \mathrm{Int}$, i.e.,

$$
E^{1}(t)=E, t \in \mathrm{Int} ; \quad E^{1}(t)=0, t \in \mathrm{I} \cup \mathrm{II} .
$$

If one represents the spinor $\psi(x)$ in the form

$$
\psi(x)=\left(\gamma^{\mu} P_{\mu}+m\right) \phi(x),
$$

where $\phi(x)$ is a new spinor, then $\phi(x)$ obeys the following equation:

$$
\left(P^{2}-m^{2}-\frac{q}{2} \sigma^{\mu \nu} F_{\mu \nu}\right) \phi(x)=0,
$$

where

$$
\begin{aligned}
& F_{\mu \nu}=\partial_{\mu} A_{\nu}-\partial_{\nu} A_{\mu}, \quad \sigma^{\mu \nu}=\frac{i}{2}\left[\gamma^{\mu}, \gamma^{\nu}\right], \\
& \frac{q}{2} \sigma^{\mu \nu} F_{\mu \nu}=i q E(t) \gamma^{0} \gamma^{1} .
\end{aligned}
$$

Solutions of the Dirac equation in a $T$-constant field were studied in detail in [24]. Below, we use these results.

First we choose a set of constant orthonormalized spinors $v_{s, r}$, with $s= \pm 1$, and $r=\left(r_{1}, r_{2}, \ldots, r_{[d / 2]-1}\right)$, $r_{i}= \pm 1$, such that $\gamma^{0} \gamma^{1} v_{s, r}=s v_{s, r}$. The indices $r_{i}$ describe the spin polarization, which is not coupled to the electric field, and together with the additional index $s$ provide a suitable parametrization of the solutions. Then we represent the spinors $\phi(x)$ as follows:

$$
\phi_{\mathbf{p}, s, r}(t, \mathbf{x})=e^{i \mathbf{p} \cdot \mathbf{x}} \varphi_{\mathbf{p}, s}(t) v_{s, r} .
$$

Thus, the time-evolution is described by the ordinary differential equation of second order

$$
\left\{\frac{d^{2}}{d t^{2}}+\left[p_{1}-q A_{1}(t)\right]^{2}+|q E| \lambda+i s q E(t)\right\} \varphi_{\mathbf{p}, s}(t)=0,
$$

where $\lambda=\left(\mathbf{p}_{\perp}^{2}+m^{2}\right) /|q E|$ and $\mathbf{p}_{\perp}$ is the transversal momentum, $\mathbf{p}_{\perp}=\left(0, p^{2}, \ldots, p^{D}\right)$.

At early $\left(t<t_{\text {in }}\right.$-region I), and late $\left(t>t_{\text {out }}\right.$-region II) times, Eq. (8) has plane wave solutions $\zeta \phi_{\mathbf{p}, s, r}$ and ${ }^{\zeta} \phi_{\mathbf{p}, s, r}$ respectively, with $\zeta= \pm 1$, which satisfy simple dispersion relations

$$
\begin{aligned}
& \mathrm{I}:{ }_{\zeta} \varphi_{\mathbf{p}, s}(t) \sim e^{-i \zeta \omega_{\text {in }} t}, \quad \text { II }:{ }^{\zeta} \varphi_{\mathbf{p}, s}(t) \sim e^{-i \zeta \omega_{\text {out }} t}, \\
& \omega_{\text {in/out }}=\sqrt{\left(p_{1}-q E t_{\text {in/out }}\right)^{2}+\mathbf{p}_{\perp}^{2}+m^{2}} .
\end{aligned}
$$

As was demonstrated in [24], the in-set $\left\{{ }_{\zeta} \psi_{\mathbf{p}, r}\right\}$ and outset $\left\{{ }^{\zeta} \psi_{\mathbf{p}, r}\right\}$ of solutions of the Dirac equation in the $T$ constant electric field can be taken in the form

$$
\begin{aligned}
& { }_{ \pm} \psi_{\mathbf{p}, r}(x)=(\gamma \cdot P+m)_{ \pm} \phi_{\mathbf{p}, \pm, r}(x), \\
& { }^{ \pm} \psi_{\mathbf{p}, r}(x)=(\gamma \cdot P+m)^{ \pm} \phi_{\mathbf{p}, \mp, r}(x) .
\end{aligned}
$$

For $t \in$ Int, the general solution of Eq. (8) is completely determined by an appropriate pair of the following linear independent Weber parabolic cylinder functions:

$$
D_{\nu-(1+s) / 2}(\zeta(1-i) \xi), \quad D_{-\nu-(1-s) / 2}(\zeta(1+i) \xi),
$$

where $\zeta= \pm, \nu=i \lambda / 2$, and

$$
\xi=\xi(t)=\frac{|q E| t-p_{1} \operatorname{sgn}(q E)}{\sqrt{|q E|}} .
$$

According to relation (2), an out-solution corresponding to a plane wave in interval II is thus described by a superposition of the Weber functions in interval Int, and extends into a superposition of particle and antiparticle $i n$-solutions in I. Then one can explicitly find the coefficients $g$. In terms of such coefficients, the differential mean number $\aleph_{\mathbf{p}, r}$ of particles created from vacuum with given $\mathbf{p}$ and $r$ at a time instant $t>t_{\text {out }}$ is

$$
\left.\aleph_{\mathbf{p}, r}=\langle 0, \text { in }| a_{\mathbf{p}, r}^{\dagger}(\text { out }) a_{\mathbf{p}, r}(\text { out }) \mid 0, \text { in }\right\rangle=\aleph_{\mathbf{p}}=\left|g\left(-\left.\right|^{+}\right)\right|^{2},
$$

where the Bogolyubov transformation between in- and out- creation and annihilation operators is used. The number of particles created is equal to the numbers of antiparticles created. Then $\aleph_{\mathbf{p}, r}$ can be treated as the number of pairs created. The result in (111) is independent of the spin polarization. That is why we use in what follows the notation $\aleph_{p}$ for the quantity (11). Note that there is no particle production after the time instant $t_{\text {out }}$. Thus, $\aleph_{\mathbf{p}}$ depends only on the interval $T$. The explicit expression for $\aleph_{\mathbf{p}}$ was studied in detail in [24]. Here we just quote the relevant results for the calculation of the vacuum mean values of the EMT and current vector.

The electric field acting during the time $T$ creates a considerable number of pairs only in a finite region in the momentum space. One can introduce a cutoff $K>>$ $\max \left\{1, m^{2} /|q E|\right\}$ such that, for $\sqrt{|q E|} T / 2>K$, one needs to consider only the region

$$
\begin{gathered}
\left|\mathbf{p}_{\perp}\right| \leq \sqrt{|q E|}[\sqrt{|q E|} T-K]^{1 / 2}, \\
-T / 2+K / \sqrt{|q E|} \leq p_{1} / q E \leq T / 2-K / \sqrt{|q E|}
\end{gathered}
$$

in the momentum space. After the cutoff, physical quantities that are expressed in terms of $\aleph_{\mathbf{p}}$ will, in general, depend on $K$. But if $T$ is big enough, so that

$$
\sqrt{|q E|} T \gg K \gg \max \left\{1, m^{2} /|q E|\right\},
$$

the dependence on $K$ can be ignored. We shall suppose that the stabilization condition (13) holds true. In this case the differential mean numbers of created pairs have the form

$$
\aleph_{\mathbf{p}} \simeq e^{-\pi \lambda}
$$

which is the same for the case of the constant uniform electric field.

Taking into account cutoff (12), we find that the total number $\aleph$ of pairs created is proportional to $d-1$ dimensional spatial volume $V_{(d-1)}$ and can be expressed through the total number density $n^{c r}$ of pairs created 
during the interval $T$ as follows:

$$
\begin{aligned}
& \aleph=\frac{V_{(d-1)}}{(2 \pi)^{d-1}} \int d \mathbf{p} \sum_{r} \aleph_{\mathbf{p}}=V_{(d-1)} n^{c r}, \\
& n^{c r}=r^{c r}\left[T+|q E|^{-1 / 2} O(K)\right], \\
& r^{c r}=\frac{2^{[d / 2]-1}}{(2 \pi)^{d-1}}|q E|^{d / 2} \exp \left(-\frac{\pi m^{2}}{|q E|}\right),
\end{aligned}
$$

where the quantity $r^{c r}$ is often called the pairs production rate.

It should be noted that the stabilization condition is written for arbitrary external field $E$. However, in what follows we are interested in strong electric fields

$$
E>E_{c} \Longrightarrow m^{2} /|q E|<1 .
$$

In what follows, when ever we speak of the strong field case we will have in mind the condition (16). In these cases the stabilization condition is simplified,

$$
\sqrt{|q E|} T>>K>>1 .
$$

The stabilization condition reveals another important dimensionless parameter $\tau$,

$$
\tau=\sqrt{|q E|} T .
$$

In the strong $T$-constant field under consideration the qualitative supposition that $T$ is big enough is equivalent to the supposition that $\tau \gg 1$. In what follows, we calculate different mean values in an approximation that is related to large $\tau$; we call such an approximation large $\tau$-limit. To explain the meaning of such a limit, we consider possible structures of the mean values that appear in our further calculations. The most general structure of the mean values has the form

$$
\langle F\rangle=\sum_{n=1}^{\infty} F_{-n} \tau^{-n}+F_{0}+\tilde{F} \ln \tau+F_{1} \tau+F_{2} \tau^{2} .
$$

The large $\tau$-limit for the mean value $\langle F\rangle$ means the leading term approximation for (19), having in mind the hierarchy $F_{0} \ll \tilde{F} \ln \tau \ll F_{1} \tau \ll F_{2} \tau^{2}$. For example, the r.h.s. in Eq. (14) represents the term $F_{0}$, while $r^{c r}$ in Eq. (15) represents $F_{1}$ in the general formula (19).

It should be noted that below we encounter cases where the large $\tau$-limit is defined by $\tau=\sqrt{|q E|} \Delta t$, with some $\Delta t$, such that $\tau \gg K \gg 1$.

It should also be noted that for large $T$, the $i n$ - and out-solutions in the interval $t \in$ Int take the form:

$$
\begin{aligned}
& { }_{+}^{-} \varphi_{\mathbf{p}, s}(t)=C D_{\nu-(1+s) / 2}( \pm(1-i) \xi), \\
& { }_{-}^{+} \varphi_{\mathbf{p}, s}(t)=C D_{-\nu-(1-s) / 2}( \pm(1+i) \xi),
\end{aligned}
$$

with the normalization constant

$$
C=(2 \pi)^{-(d-1) / 2}|2 q E|^{-1 / 2} \exp (-\pi \lambda / 8) .
$$

It is supposed that the measurement is carried out at some time after switching off the electric field, i.e., decoherence occurs after the electric field is switched off. Of course, one can consider the case when decoherence occurs earlier, for example, at an instant $t_{d e c}$, $t_{\text {in }}<t_{\text {dec }}<t_{\text {out }}$. Let us suppose, for example, that the interval $t_{d e c}-t_{\text {in }}$ satisfies stabilization conditions similar to (13), $\sqrt{|q E|}\left(t_{d e c}-t_{\text {in }}\right) \gg K$. Then the differential mean number of pairs created in the large $t_{d e c}-t_{i n}$ limit, when

$$
\begin{gathered}
\left|\mathbf{p}_{\perp}\right| \leq \sqrt{|q E|}\left[\sqrt{|q E|}\left(t_{d e c}-t_{i n}\right)-K\right]^{1 / 2}, \\
t_{i n}+K / \sqrt{|q E|} \leq p_{1} / q E \leq t_{d e c}-K / \sqrt{|q E|},
\end{gathered}
$$

is given by (14) and the total number density of particles created is $r^{c r}\left(t_{d e c}-t_{i n}\right)$. For the time of decoherence $t_{d e c}$, which is sufficiently close to $t_{\text {out }},\left(t_{\text {out }}-t_{\text {dec }}\right) / T \ll$ 1 , the difference of this density from the density $n^{c r}$ can be ignored and the interpretation of particles at $t_{d e c}$ as final out-particles already makes sense.

However, if the interval $t_{\text {out }}-t_{\text {dec }}$ is comparable with the interval $t_{d e c}-t_{i n}$ then it is necessary to consider the further evolution of many-particle state given initially by the distribution (14) at the time $t_{d e c}$, which is a completely different task, see for example [25]. In this case, the differential mean numbers of additional pairs created by the external field during the interval from $t_{d e c}$ to $t_{\text {out }}$ is given by Eq. (59) of the work 25] as follows:

$$
\Delta N_{\mathbf{p}}=\aleph_{\mathbf{p}}\left[1-\left(N_{\mathbf{p}}^{(+)}(i n)+N_{\mathbf{p}}^{(-)}(i n)\right)\right],
$$

where $N_{\mathbf{p}}^{(+)}(i n)=N_{\mathbf{p}}^{(-)}(i n)=\aleph_{\mathbf{p}}$ are differential mean numbers of initial particles and antiparticles at $t_{d e c}$, given by Eq. (14). If $N_{\mathbf{p}}^{(+)}(i n)+N_{\mathbf{p}}^{(-)}(i n)>1$ then $\Delta N_{\mathbf{p}}$ is negative, that is, the annihilation of the existing pairs of particles and antiparticles results from the electric field. Integrating and summing distribution (23) over the quantum numbers under conditions (22), we obtain the additional number density of pairs

$$
\Delta n^{c r}=\left[1-2^{2-d / 2} \exp \left(-\frac{\pi m^{2}}{|q E|}\right)\right] r^{c r}\left(t_{d e c}-t_{i n}\right) .
$$

Summing the density of pairs created at $t_{d e c}$, $r^{c r}\left(t_{d e c}-t_{i n}\right)$, the additional density $\Delta n^{c r}$, and the number density of pairs created from vacuum states after $t_{d e c}, r^{c r}\left(t_{\text {out }}-t_{d e c}\right)$, we find the following total number density of pairs created during the interval $T$,

$$
n^{c r}\left(t_{\text {out }}-t_{\text {dec }} \mid t_{d e c}-t_{\text {in }}\right)=n^{c r}+\Delta n^{c r} .
$$

Thus, if decoherence occurs at $t_{d e c}$, the final number density of pairs at the time instant $t_{\text {out }}$ differs from $n^{\text {cr }}$ by $\Delta n^{c r}$. In the case of strong electric field, $\frac{m^{2}}{|q E|} \ll 1$, $\Delta n^{c r} \approx 0$ for $d=4, \Delta n^{c r}<0$ for $d=2,3$, and $\Delta n^{c r}>0$ for $d>4$. We see that the intermediate decoherence at the early time significantly reduces the measured density of final particles in low-dimensional systems, and increases in high-dimensional systems. Decoherence can 
occur once or many times during the interval $T$. The choice of a suitable model of decoherence depends on the physical nature of the phenomenon.

It is useful to comment the case when the initial manyparticle state is the thermodynamical equilibrium of noninteracting particles at the temperature $\Theta$ with the chemical potential $\mu_{c h}$,

$$
N_{\mathbf{p}}^{( \pm)}(i n)=\left\{\exp \left[\left(\omega_{i n}-\mu_{c h}\right) / \Theta\right]+1\right\}^{-1},
$$

where $\omega_{\text {in }}$ is given by Eq. (9). Then the differential mean number $\Delta N_{\mathbf{p}}$ of additional pairs created by the external field during the interval $T$, has the form (23), where $\aleph_{\mathbf{p}}$ is given by (14). Taking into account the cutoff (12), one can find the total number of additional particles created. In consequence, one can see that at low temperatures, $\left(m-\mu_{c h}\right) / \Theta \gg 1$, such a total number differs from the zero-temperature result (15) by a next-to-leading term that is not essential in the large $\tau$-limit, see [25]. We consider the system at high temperatures when all the energies of the created and accelerated particles are much lower than the temperature $\Theta$, hence $|q E| T / \Theta \ll 1$. One can extract from results of the work [25], that the total number of additional particles created under such a condition is much less than that in (15). However, we stress that at high temperatures, the polarization effect in the current and energy densities of the initial gas of charged particles given by Eq. (127) in [10], is much stronger than effect from the pair creation given by Eq. (72) in [10]. This could lead to screening the electric field before the effects of pair creation manifest themselves. One can see that the vacuum contributions dominate in comparison with contributions due to the low temperature and particle density in the initial state. That is why we restrict ourselves to the case of the vacuum initial state.

\section{ENERGY-MOMENTUM TENSOR AND CURRENT VECTOR}

\section{A. Observables and Green functions}

We consider here various singular functions of the Dirac field. With the help of these functions, different physical quantities can be calculated. For example, mean values (3) can be calculated with the help of the so-called in-propagator:

$$
S_{i n}^{c}\left(x, x^{\prime}\right)=i\left\langle 0, i n\left|T \Psi(x) \bar{\Psi}\left(x^{\prime}\right)\right| 0, i n\right\rangle .
$$

In turn, this propagator can be determined via the $i n$ solutions as follows

$$
\begin{aligned}
& S_{i n}^{c}\left(x, x^{\prime}\right)=\theta\left(t-t^{\prime}\right) S_{i n}^{-}\left(x, x^{\prime}\right)-\theta\left(t^{\prime}-t\right) S_{i n}^{+}\left(x, x^{\prime}\right), \\
& S_{i n}^{\mp}\left(x, x^{\prime}\right)=i \int d \mathbf{p} \sum_{r} \pm \psi_{\mathbf{p}, r}(x)_{ \pm} \bar{\psi}_{\mathbf{p}, r}\left(x^{\prime}\right) .
\end{aligned}
$$

Using relation (2) and properties of the $g$ coefficients, one can divide the propagator (25) into a sum of two terms,

$$
S_{i n}^{c}\left(x, x^{\prime}\right)=S^{c}\left(x, x^{\prime}\right)+S^{p}\left(x, x^{\prime}\right),
$$

where the first term is the causal (Feynman) propagator,

$$
\begin{aligned}
& S^{c}\left(x, x^{\prime}\right)=i\left\langle 0, \text { out }\left|T \Psi(x) \bar{\Psi}\left(x^{\prime}\right)\right| 0, \text { in }\right\rangle c_{v}^{-1}, \\
& \left.c_{v}=\langle 0, \text { out }| 0, \text { in }\right\rangle .
\end{aligned}
$$

Here $c_{v}$ is the vacuum-to-vacuum probability amplitude. This propagator can be represented as follows:

$$
\begin{aligned}
& S^{c}\left(x, x^{\prime}\right)=\theta\left(t-t^{\prime}\right) S^{-}\left(x, x^{\prime}\right)-\theta\left(t^{\prime}-t\right) S^{+}\left(x, x^{\prime}\right), \\
& S^{-}\left(x, x^{\prime}\right)=i \int d \mathbf{p} \sum_{r}{ }^{+} \psi_{\mathbf{p}, r}(x) g\left(+^{+}\right)^{-1}+\bar{\psi}_{\mathbf{p}, r}\left(x^{\prime}\right), \\
& S^{+}\left(x, x^{\prime}\right)=i \int d \mathbf{p} \sum_{r}-\psi_{\mathbf{p}, r}(x)\left[g\left(-\left.\right|^{-}\right)^{-1}\right]^{*}-\bar{\psi}_{\mathbf{p}, r}\left(x^{\prime}\right) .
\end{aligned}
$$

The second term in (26) has the following form:

$$
\begin{aligned}
S^{p}\left(x, x^{\prime}\right)=i \int & d \mathbf{p} \sum_{r}-\psi_{\mathbf{p}, r}(x) \\
& \times\left[g\left(+\left.\right|^{-}\right) g\left(-\left.\right|^{-}\right)^{-1}\right]^{*}+\bar{\psi}_{\mathbf{p}, r}\left(x^{\prime}\right) .
\end{aligned}
$$

The current density, $j_{c r}^{\mu}(t)$, and EMT, $T_{\mu \nu}^{c r}(t)$, of created particles at $t \gtrsim t_{\text {out }}$, are expressed via the mean values of the normal form of $j^{\mu}$ and $T_{\mu \nu}$ operators with respect to the out-vacuum. Namely,

$$
\begin{aligned}
& j_{c r}^{\mu}(t)=\left\langle j^{\mu}(t)\right\rangle-\left\langle j^{\mu}(t)\right\rangle_{\text {out }}, \\
& \left\langle j^{\mu}(t)\right\rangle_{\text {out }}=\left\langle 0, \text { out }\left|j^{\mu}\right| 0, \text { out }\right\rangle, \\
& T_{\mu \nu}^{c r}(t)=\left\langle T_{\mu \nu}(t)\right\rangle-\left\langle T_{\mu \nu}(t)\right\rangle_{\text {out }}, \\
& \left\langle T_{\mu \nu}(t)\right\rangle_{\text {out }}=\left\langle 0, \text { out }\left|T_{\mu \nu}\right| 0, \text { out }\right\rangle .
\end{aligned}
$$

These mean values can be calculated with the help of the so-called out-propagator

$$
S_{\text {out }}^{c}\left(x, x^{\prime}\right)=i\left\langle 0, \text { out }\left|T \Psi(x) \bar{\Psi}\left(x^{\prime}\right)\right| 0, \text { out }\right\rangle .
$$

Similarly to the case of the in-propagator, one can relate this propagator to $S^{c}\left(x, x^{\prime}\right)$ as follows:

$$
S_{\text {out }}^{c}\left(x, x^{\prime}\right)=S^{c}\left(x, x^{\prime}\right)+S^{\bar{p}}\left(x, x^{\prime}\right),
$$

where

$$
\begin{aligned}
S^{\bar{p}}\left(x, x^{\prime}\right)=-i \int & d \mathbf{p} \sum_{r}+\psi_{\mathbf{p}, r}(x) \\
& \times g\left(+\left.\right|^{+}\right)^{-1} g\left(+\left.\right|^{-}\right)-\bar{\psi}_{\mathbf{p}, r}\left(x^{\prime}\right) .
\end{aligned}
$$

The quantities (3) and (30) are real valued and can be represented as:

$$
\begin{aligned}
& \left\langle j^{\mu}(t)\right\rangle=\operatorname{Re}\left\langle j^{\mu}(t)\right\rangle^{c}+\operatorname{Re}\left\langle j^{\mu}(t)\right\rangle^{p} \\
& \left\langle T_{\mu \nu}(t)\right\rangle=\operatorname{Re}\left\langle T_{\mu \nu}(t)\right\rangle^{c}+\operatorname{Re}\left\langle T_{\mu \nu}(t)\right\rangle^{p} \\
& \left\langle j^{\mu}(t)\right\rangle_{\text {out }}=\operatorname{Re}\left\langle j^{\mu}(t)\right\rangle^{c}+\operatorname{Re}\left\langle j^{\mu}(t)\right\rangle^{\bar{p}} \\
& \left\langle T_{\mu \nu}(t)\right\rangle_{\text {out }}=\operatorname{Re}\left\langle T_{\mu \nu}(t)\right\rangle^{c}+\operatorname{Re}\left\langle T_{\mu \nu}(t)\right\rangle^{\bar{p}}
\end{aligned}
$$


where

$$
\begin{aligned}
& \left\langle j^{\mu}(t)\right\rangle^{c, p, \bar{p}}=\left.i q \operatorname{tr}\left[\gamma^{\mu} S^{c, p, \bar{p}}\left(x, x^{\prime}\right)\right]\right|_{x=x^{\prime}}, \\
& \left\langle T_{\mu \nu}(t)\right\rangle^{c, p, \bar{p}}=\left.i \operatorname{tr}\left[A_{\mu \nu} S^{c, p, \bar{p}}\left(x, x^{\prime}\right)\right]\right|_{x=x^{\prime}}, \\
& A_{\mu \nu}=1 / 4\left[\gamma_{\mu}\left(P_{\nu}+P_{\nu}^{\prime *}\right)+\gamma_{\nu}\left(P_{\mu}+P_{\mu}^{\prime *}\right)\right] .
\end{aligned}
$$

Here tr stands for the trace in the $\gamma$-matrices indices and the limit $x \rightarrow x^{\prime}$ is understood as follows:

$$
\begin{aligned}
\operatorname{tr}\left[\cdots\left(x, x^{\prime}\right)\right]_{x=x^{\prime}}=\frac{1}{2} & {\left[\lim _{t \rightarrow t^{\prime}-0} \operatorname{tr}\left[\cdots\left(x, x^{\prime}\right)\right]\right.} \\
& \left.\left.+\lim _{t \rightarrow t^{\prime}+0} \operatorname{tr}\left[\cdots\left(x, x^{\prime}\right)\right]\right]_{\mathbf{x}=\mathbf{x}^{\prime}}\right] .
\end{aligned}
$$

The representations (33) and (34) imply that

$$
\begin{aligned}
& j_{c r}^{\mu}(t)=\operatorname{Re}\left\langle j^{\mu}(t)\right\rangle^{p}-\operatorname{Re}\left\langle j^{\mu}(t)\right\rangle^{\bar{p}} \\
& T_{\mu \nu}^{c r}(t)=\operatorname{Re}\left\langle T_{\mu \nu}(t)\right\rangle^{p}-\operatorname{Re}\left\langle T_{\mu \nu}(t)\right\rangle^{\bar{p}}
\end{aligned}
$$

Note that the mean current $\left\langle j^{\mu}(t)\right\rangle$ and the physical part of the mean value $\left\langle T_{\mu \nu}(t)\right\rangle$ are zero for $t<t_{i n}$, when the electric field is zero. We are only interested in these mean values for large $T$ and for $t \in I n t$, when the time $t$ from the latter interval is sufficiently large,

$$
\sqrt{|q E|}\left(t-t_{\text {in }}\right) \gg K \gg \max \left\{1, m^{2} /|q E|\right\},
$$

where $K$ is the cutoff introduced before in Eq. (22). Then it is sufficient to use the in- and out-solutions in asymptotic form (20) for the functions $S^{c, p}\left(x, x^{\prime}\right)$ defined above. At late times, $t>t_{\text {out }}$, the solutions ${ }^{ \pm} \psi_{\mathbf{p}, r}(x)$ reduce to free plane waves in accordance with Eq. (9).

Some components of $\left\langle j^{\mu}(t)\right\rangle^{c, p, \bar{p}}$ and $\left\langle T_{\mu \nu}(t)\right\rangle^{c, p, \bar{p}}$ are finite (do not have any $T$-divergences) as $T \rightarrow \infty$. In the expressions for these components, we can use asymptotic (as $T \rightarrow \infty$ ) forms of the singular functions $S^{c, p, \bar{p}}\left(x, x^{\prime}\right)$, that is, the functions $S^{c, p, \bar{p}}\left(x, x^{\prime}\right)$ in the constant electric field. For such singular functions, the so-called FockSchwinger proper time representations hold true [26] (it should be noted that the functions $S^{p}\left(x, x^{\prime}\right)$ and $S^{\bar{p}}\left(x, x^{\prime}\right)$ defined above coincide with the functions $-S^{a}\left(x, x^{\prime}\right)$ and $-S^{p}\left(x, x^{\prime}\right)$ respectively, used in the article [26]). In the Fock-Schwinger representations the causal propagator $S^{c}\left(x, x^{\prime}\right)$ defined by Eq. (28), $S^{p}\left(x, x^{\prime}\right)$ in (29), and $S^{\bar{p}}\left(x, x^{\prime}\right)$ in (32) have the following integral representa- tions:

$$
\begin{aligned}
S^{c, p, \bar{p}}\left(x, x^{\prime}\right)= & (\gamma P+m) \Delta^{c, p, \bar{p}}\left(x, x^{\prime}\right), \\
\Delta^{c}\left(x, x^{\prime}\right)= & \int_{\Gamma_{c}} d s f\left(x, x^{\prime}, s\right), \\
\Delta^{p}\left(x, x^{\prime}\right)=- & \int_{\Gamma_{p}} d s f\left(x, x^{\prime}, s\right) \\
& \quad-\Theta\left(x_{1}-x_{1}^{\prime}\right) \int_{\Gamma_{3}+\Gamma_{2}-\Gamma_{p}} d s f\left(x, x^{\prime}, s\right), \\
\Delta^{\bar{p}}\left(x, x^{\prime}\right)=- & \int_{\Gamma_{p}} d s f\left(x, x^{\prime}, s\right) \\
& \quad-\Theta\left(x_{1}^{\prime}-x_{1}\right) \int_{\Gamma_{3}+\Gamma_{2}-\Gamma_{p}} d s f\left(x, x^{\prime}, s\right),
\end{aligned}
$$

where the Fock-Schwinger kernel $f\left(x, x^{\prime}, s\right)$ reads:

$$
\begin{aligned}
f\left(x, x^{\prime}, s\right)= & \exp \left(-i \frac{q}{2} \sigma^{\mu \nu} F_{\mu \nu} s\right) f^{(0)}\left(x, x^{\prime}, s\right), \\
f^{(0)}\left(x, x^{\prime}, s\right)= & -\frac{1}{(4 \pi i)^{d / 2}} \frac{q E s^{-d / 2+1}}{\sinh (q E s)} e^{i q \Lambda} e^{-i m^{2} s} \\
& \times \exp \left[\frac{1}{4 i}\left(x-x^{\prime}\right) q F \operatorname{coth}(q F s)\left(x-x^{\prime}\right)\right],
\end{aligned}
$$

$\operatorname{coth}(q F s)$ is the matrix with the components $[\operatorname{coth}(q F s)]_{\nu}^{\mu}$, and $\Lambda=\left(x_{0}+x_{0}^{\prime}\right)\left(x_{1}-x_{1}^{\prime}\right) E / 2$. All integration contours in the $s$-complex plane are shown in Fig. 1 The integral along the contour $\Gamma_{c}$, that is, along the real positive semiaxis, corresponds to the well-known Schwinger's representation of the Feynman propagator.

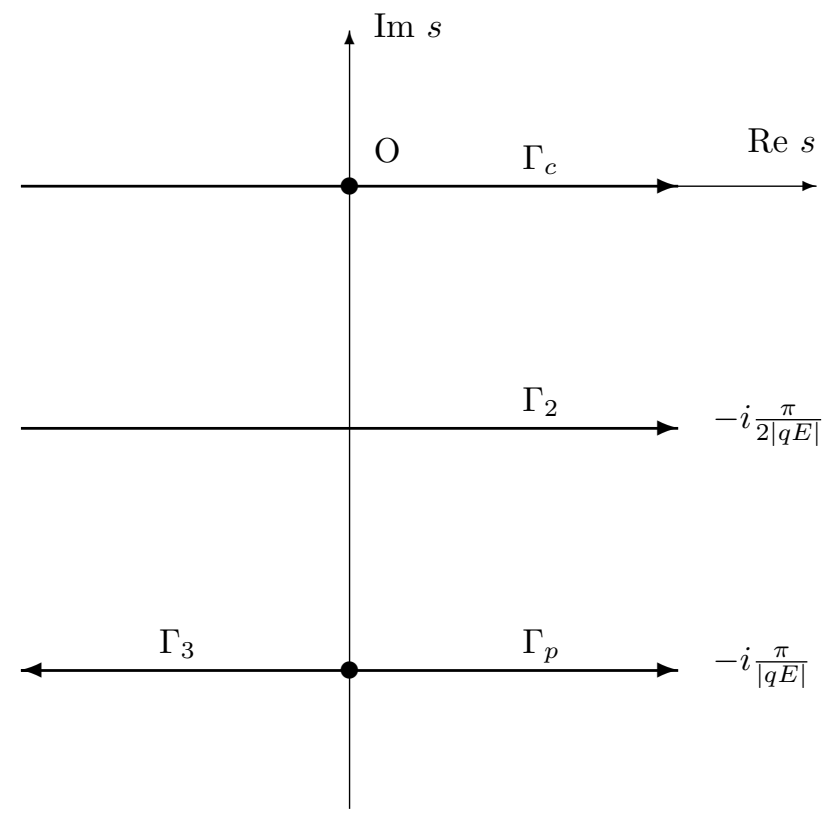

FIG. 1. Contours of integration $\Gamma_{2}, \Gamma_{3}, \Gamma_{c}, \Gamma_{p}$. 
The only singular points of the kernel $f\left(x, x^{\prime}, s\right)$ in the lower half-plane outside of the origin are $s_{n}=$ $-i \pi n /|q E|, n=1,2, \ldots$

\section{B. Peculiarities in odd dimensions}

We shall see that in odd dimensions there exist current components related to the so-called Chern-Simons term of the effective action. The components $\left\langle j^{1}(t)\right\rangle^{p, \bar{p}}$ are ill defined in the proper time representation due to the divergence in the limit $T \rightarrow \infty$. They will be treated in detail at finite $T$ in Subsection IIE All other current components $\left\langle j^{\mu}(t)\right\rangle^{c, p, \bar{p}}$ are finite as $T \rightarrow \infty$ and can be determined with the help of Eqs. (38). We first consider the case of $d=3$ dimensions. In this case, there are two non-equivalent representations for the $\gamma$-matrices,

$$
\gamma^{0}=\sigma^{3}, \gamma^{1}=i \sigma^{2}, \gamma^{2}=-i( \pm 1) \sigma^{1},
$$

where $\sigma^{i}$ are the Pauli matrices and the signs \pm 1 correspond to different fermion species, which we call as \pm -fermions, respectively. By using the formula

$$
\exp \left(-i \frac{q}{2} \sigma^{\mu \nu} F_{\mu \nu} s\right)=\cosh (q E s)+\gamma^{0} \gamma^{1} \sinh (q E s),
$$

one finds that

$\operatorname{tr}\left[\gamma^{\mu} m f(x, x, s)\right]=-i( \pm 1) 2 m \sinh (q E s) f^{(0)}(x, x, s) \delta_{\mu, 2}$.

Inserting this expression and $f^{(0)}\left(x, x^{\prime}, s\right)$ given by Eq. (39) into Eqs. (38) and taking into account that the integral along the contour $\Gamma_{3}+\Gamma_{2}-\Gamma_{p}$ does not contribute to quantities given by Eqs. (35), we obtain the following result:

$$
\begin{aligned}
& \left\langle j^{\mu}(t)\right\rangle^{c}= \pm \delta_{\mu, 2} \frac{e^{2} E}{4 \pi}, \quad\left\langle j^{0}(t)\right\rangle^{p, \bar{p}}=0, \\
& \left\langle j^{2}(t)\right\rangle^{p, \bar{p}}=\mp \frac{e^{2} E}{4 \pi^{3 / 2}} \Gamma\left(\frac{1}{2}, \frac{\pi m^{2}}{|q E|}\right),
\end{aligned}
$$

where $\Gamma(1 / 2, x)$ is the incomplete gamma function. In $d \neq 3$ dimensions we find that

$$
\left\langle j^{\mu}(t)\right\rangle^{c}=0, \forall \mu ; \quad\left\langle j^{\mu}(t)\right\rangle^{p, \bar{p}}=0, \mu \neq 1 .
$$

We see that in $d=3$ dimensions there are nonzero components $\left\langle j^{\mu}(t)\right\rangle_{\perp}^{c, p, \bar{p}}=\delta_{\mu, 2}\left\langle j^{2}(t)\right\rangle^{c, p, \bar{p}}$ that are orthogonal to the electric field direction. By covariance, one concludes from Eq. (41) that the component $\left\langle j^{\mu}(t)\right\rangle^{c}$ in an arbitrary inertial reference frame is

$$
\left\langle j^{\mu}(t)\right\rangle^{c}= \pm \frac{e^{2}}{8 \pi} e^{\mu \alpha \beta} F_{\alpha \beta}
$$

for any electric-like field (i.e., when the magnetic field can be removed by a Lorentz transformation). This expression has previously been obtained for the magnetic-like uniform electromagnetic field and related to the additional Chern-Simons term in the Euler-Heisenberg effective action,

$$
\Gamma_{C S}= \pm \frac{e^{2}}{16 \pi} \int d t d \mathbf{x} e^{\mu \alpha \beta} F_{\alpha \beta} A_{\mu},
$$

in [27, 28], while the expression for $\left\langle j^{\mu}(t)\right\rangle_{\perp}^{p, \bar{p}}$ that can be extracted from Eq. (41) is new. This radiatively induced term $\Gamma_{C S}$ is the topological mass term (ChernSimons invariant). The Chern-Simons term $\Gamma_{C S}$ formally vanishes for a uniform field, but its variation with respect to $A_{\mu}$ produces a nonvanishing current $\left\langle j^{\mu}(t)\right\rangle^{c}$. For the case of magnetic-like field, i.e., when the electric field can be removed by a Lorentz transformation, one has $\left\langle j^{\mu}(t)\right\rangle=\left\langle j^{\mu}(t)\right\rangle^{c}$ and $\left\langle j^{\mu}(t)\right\rangle_{\perp}^{p, \bar{p}}=0$. However, in the case of an electric-like field under consideration, we see that there is an additional term $\left\langle j^{2}(t)\right\rangle^{p, \bar{p}} \neq 0$ given by Eq. (41). This is what distinguishes substantially the case of electric-like field from the case of magnetic-like field. Thus, we see that the Chern-Simons term is present in a properly regularized effective action for the electriclike field with odd number of fermion species. The signs " \pm " in Eq. (41) are opposite for each of the two possible fermion species. The absence of similar components in expressions (42) in higher $(d>3)$ odd dimensions is related to the fact that we consider a special case where the magnetic field is absent. Note that in arbitrary constant field such current components are orthogonal to the electric and magnetic field, $\left\langle j^{\mu}(t)\right\rangle_{\perp}^{c, p, \bar{p}} F_{\mu \nu}=0$, and proportional to the product of all eigenvalues of the field tensor $F_{\mu \nu}$, see the appropriate expression for $\operatorname{tr}\left[\gamma^{\mu} m f(x, x, s)\right]$ in [26]. Separating the transverse components, we represent the vacuum current density in the final form

$$
\begin{aligned}
& \left\langle j^{\mu}(t)\right\rangle=\left\langle j^{\mu}(t)\right\rangle_{\perp}+\operatorname{Re}\left\langle j^{\mu}(t)\right\rangle_{\|}^{p}, \\
& \left\langle j^{\mu}(t)\right\rangle_{\perp}=\left\langle j^{\mu}(t)\right\rangle_{\perp}^{c}+\left\langle j^{\mu}(t)\right\rangle_{\perp}^{p},
\end{aligned}
$$

where finite quantities $\left\langle j^{\mu}(t)\right\rangle_{\perp}^{c, p}$ are given by Eqs. (41) and (42), whereas the component $\left\langle j^{\mu}(t)\right\rangle_{\|}^{p}=\delta_{\mu, 1}\left\langle j^{1}(t)\right\rangle^{p}$ directed along the electric field must be treated for finite $T$. One can see from Eqs. (36) and (41) that the transverse current of created particles is absent, $j_{c r}^{2}(t)=0$, and this conclusion holds true for arbitrary constant field in higher odd dimensions. Then the term $\left\langle j^{\mu}(t)\right\rangle_{\perp}$ represents transverse vacuum-polarization current, in particular for $d=3$ it has the form

$$
\left\langle j^{\mu}(t)\right\rangle_{\perp}= \pm \delta_{\mu, 2} \frac{e^{2}}{4 \pi^{3 / 2}} \gamma\left(\frac{1}{2}, \frac{\pi m^{2}}{|q E|}\right) E,
$$

where $\gamma(1 / 2, x)$ is the incomplete gamma function.

The factor in front of $E$ in Eq. (44) can be considered as the nonequilibrium Hall conductivity for large duration of electric field $\Delta t$ satisfying condition (37). It should be noted that the expression (44) is close to the one given by Eq. (7) in [29]. The latter was obtained in the framework of one-particle WKB calculations, using an analogy with the Landau-Zener tunneling. Note that 
it is quite difficult in the framework of the one-particle theory to distinguish between the current of real particles, which remains after switching off of the electric field, and the pure vacuum-polarization current, which disappears in this case, although there is no dissipation in the model. We stress that the latter current vanishes together with the electric field. Therefore, in this problem, a use of the analogy with the tunneling, i.e., with Schwinger's pair-production rate, should be justified. In fact, our exact result (44), obtained in the framework of $\mathrm{QED}$, is such a justification of the one-particle calculation [29], at the same time it shows the limits of its validity, namely, it holds true only before the switching off the electric field. The quantity $\left\langle j^{\mu}(t)\right\rangle_{\perp}^{p, \bar{p}}$ is exponentially small for weak electric fields, $m^{2} /|q E| \gg 1$, in which case $\left\langle j^{2}(t)\right\rangle \approx\left\langle j^{2}(t)\right\rangle^{c}$. On the other hand, in the strong-field limit, $m^{2} /|q E| \ll 1$, both contributions are comparable, $\left\langle j^{2}(t)\right\rangle^{p} \approx-\left\langle j^{2}(t)\right\rangle^{c}$, and then

$$
\left\langle j^{2}(t)\right\rangle \approx \pm \frac{e^{3 / 2} E m}{2 \pi \sqrt{|E|}}
$$

The conductivity that follows from eq. (45) coincides with the one given by Eq. (9) in [29].

As it is known, the term $\left\langle j^{2}(t)\right\rangle^{c}$ is related to the standard effective action and therefore to probability amplitudes of processes, while the term $\left\langle j^{2}(t)\right\rangle$ presents a contribution to mean values which, in general, are quite different from such amplitudes. We see that $\left\langle j^{2}(t)\right\rangle^{c}$ and the probability amplitudes remain unchanged as $m \rightarrow 0$. In contrast to the behavior of quantity $\left\langle j^{2}(t)\right\rangle^{c}$, the mean value $\left\langle j^{2}(t)\right\rangle$ tends to 0 as $m \rightarrow 0$. It is important to note that these constant values of both $\left\langle j^{\mu}(t)\right\rangle_{\perp}^{c}$ and $\left\langle j^{\mu}(t)\right\rangle_{\perp}^{p}$ are obtained in the limit $T \rightarrow \infty$, that is, Eqs. (41) hold true for instants of time $t$ before the electric field is switched off. At early, $t<t_{\text {in }}$, and late times, $t>t_{\text {out }}$, the $T$-constant electric field is absent, and one can see from the exact formula (35) that the vacuum polarization current vanishes in this case, $\left\langle j_{\mu}(t)\right\rangle^{c}=\left\langle j^{\mu}(t)\right\rangle_{\perp}^{p}=0$. These results can be generalized to the case of massless fermions in an electric-like constant electromagnetic field in higher odd dimensions, when the electric field cannot be removed by the Lorentz transformation and all eigenvalues of the field tensor $F_{\mu \nu}$ are different from zero. In this case when $t<t_{\text {out }}$, the transverse component of vacuum polarization current $\left\langle j^{\mu}(t)\right\rangle_{\perp}^{c}$ is nonzero, however, the total transverse mean value $\left\langle j^{\mu}(t)\right\rangle_{\perp}$ is equal to zero. Thus, the vacuum polarization in the electric field in odd dimensions is qualitatively different for mean values and amplitudes of processes. This is what distinguishes substantially the case of electric-like field from the case of magnetic-like field.

\section{EMT}

Using representations (35) and (38), we obtain components of the EMT as follows:

$$
\begin{aligned}
& \left\langle T_{\mu \nu}(t)\right\rangle^{c, p, \bar{p}}=0, \quad \mu \neq \nu, \\
& \operatorname{Re}\left\langle T_{00}(t)\right\rangle^{c}=-\operatorname{Re}\left\langle T_{11}(t)\right\rangle^{c}=E \frac{\partial \operatorname{Re} \mathcal{L}(t)}{\partial E}-\operatorname{Re} \mathcal{L}(t), \\
& \operatorname{Re}\left\langle T_{i i}(t)\right\rangle^{c}=\operatorname{Re} \mathcal{L}(t), \quad i=2,3, \ldots, D,
\end{aligned}
$$

where

$$
\begin{aligned}
& \mathcal{L}=\frac{1}{2} \int_{0}^{\infty} \frac{d s}{s} \operatorname{tr} f(x, x, s), \\
& \operatorname{tr} f(x, x, s)=2^{[d / 2]} \cosh (q E s) f^{(0)}(x, x, s) .
\end{aligned}
$$

It should be noted that the diagonal elements $\left\langle T_{\mu \mu}(t)\right\rangle^{p}$ are ill defined in the proper time representation due to the divergence at the limit $T \rightarrow \infty$ and will be treated at finite $T$ in Subsec. IIIE, The quantity $\mathcal{L}$ in (48) can be identified as the non-renormalized one-loop effective Euler-Heisenberg Lagrangian of the Dirac field in an uniform electric field. It is the density of the one-loop effective action $W$,

$$
W=\int \mathcal{L} d t d \mathbf{x}=-i \ln c_{v} .
$$

that is defined in general via the vacuum-to-vacuum amplitude (27). Its imaginary part represents the vacuumto-vacuum probability, as follows:

$$
\left|c_{v}\right|^{2}=e^{-2 \operatorname{Im} W} .
$$

It is a global physical quantity. It is free of ultraviolet divergences, because creation of pairs with infinitely large momenta is suppressed. The Bogolyubov transformation between in- and out- creation and annihilation operators allows one to relate $c_{v}$ with the $g$ coefficients from (2) [1, 25]:

$$
c_{v}=\exp \left\{\operatorname{tr} \ln g\left(-\left.\right|^{-}\right)^{*}\right\} .
$$

For the $T$-constant field, when $T$ satisfies the stabilization conditions (13), the probability (50) can be expressed via total number of particles created (15) in the following form:

$$
\ln \left|c_{v}\right|^{2}=-\rho \aleph, \quad \rho=\sum_{n=0}^{\infty}(n+1)^{-d / 2} \exp \left(-n \pi m^{2} /|q E|\right),
$$

see [24]. It follows from (51) that $\aleph \sim T V_{(d-1)}$, then $\ln \left|c_{v}\right|^{2}$ is proportional to the spatial volume $V_{(d-1)}$ and the field duration $T$. It can be verified that the result (51) can be obtained with the help of $\operatorname{Im} \mathcal{L}$ (48), provided that the integral over the time $t$ is identified with the field duration $T, \int d t=T,[26$. Thus, $\operatorname{Im} W$ is finite for finite values of $V_{(d-1)}$ and $T$. Note that in the strong-field case, $m^{2} /|q E| \ll 1$, for $d \geq 3$, the leading term in the r.h.s. of 
(51) is the ordinary Riemann zeta function, $\rho=\zeta_{R}(d / 2)$. For $d=2$, the leading term is $\rho=\ln \left(|q E| / m^{2}\right)$. That means that in $d=2$ dimensions the vacuum-to-vacuum probability of massless fermions in quasiconstant electric field is ill defined. In this case, the mass term $m \neq 0$ has to be considered as an infrared cutoff.

Both the vacuum mean values of the total current density and of the EMT are represented as sums of two contributions: $\operatorname{Re}\left\langle j^{\mu}(t)\right\rangle^{c}$ (resp. $\operatorname{Re}\left\langle T_{\mu \nu}(t)\right\rangle^{c}$ and $\operatorname{Re} \mathcal{L}(t)$ ) that can be associated with the vacuum polarization, while $\left\langle j^{\mu}(t)\right\rangle^{p}=j_{c r}^{\mu}(t)+\left\langle j^{\mu}(t)\right\rangle^{\bar{p}}$ (resp. $\left\langle T_{\mu \nu}(t)\right\rangle^{p}=$ $\left.T_{\mu \nu}^{c r}(t)+\left\langle T_{\mu \nu}(t)\right\rangle^{\bar{p}}\right)$ can be associated with the pair creation due to the term $j_{c r}^{\mu}(t)$ (resp. $\left.T_{\mu \nu}^{c r}(t)\right)$. The latter follows from the fact that $S^{p}\left(x, x^{\prime}\right)$ with all its derivatives, given by Eq. (29), is exponentially small due to the smallness of $\left|g\left(+\left.\right|^{-}\right)\right|$, when the electric field is weak, $m^{2} /|q E| / \gg 1$. Of course, in general, such unambiguous division of physical quantities due to particle creation and the vacuum polarization is not possible. However, it can be done in some specific cases and for some specific quantities. It is clear that $j_{c r}^{\mu}(t)$ and $T_{\mu \nu}^{c r}(t)$ depend on the history of the process and retain their latest values at $t>t_{\text {out }}$. On the other hand, we see from Eqs. (41) and (47) that the nonzero real parts of the quantities $\left\langle j^{\mu}(t)\right\rangle^{c}, \operatorname{Re}\left\langle T_{\mu \nu}(t)\right\rangle^{c}$, and $\left\langle j^{\mu}(t)\right\rangle_{\perp}^{p}$ are time-independent for the time $t \in$ Int. At early $t<t_{\text {in }}$ and late times $t>t_{\text {out }}$, we have $\left\langle j^{\mu}(t)\right\rangle^{c}=\left\langle j^{\mu}(t)\right\rangle_{\perp}^{p}=0$ and the mean $\left\langle T_{\mu \nu}(t)\right\rangle^{c}$ is reduced to its free value for $E=0$. Thus, we see that $\left\langle j^{\mu}(t)\right\rangle^{c},\left\langle j^{\mu}(t)\right\rangle_{\perp}^{p}$, and $\operatorname{Re}\left\langle T_{\mu \nu}(t)\right\rangle^{c}$ depend on the electric field at the time $t$, but do not depend on the history of the process, that is, they are local quantities and represent the vacuum polarization contribution. We continue to study each of these local and nonlocal terms independently in the following sections.

\section{Mean values: renormalization}

The integrals (47) are divergent due to the real part of the effective Lagrangian (48) which is ill defined. This real part must be regularized and renormalized. In low dimensions, $d \leq 4$, Re $\mathcal{L}$ can be regularized in the propertime representation and renormalized by the Schwinger renormalizations of the charge and the electromagnetic field [30]. In higher dimensions, a different approach is required. Note that in the case of $d>4$ dimensions, plane QED is rather unrealistic system, however, it is common to use it as a simple (Abelian) model to consider the qualitative behavior of a quantum gauge field theory as a function of $d$, see, for example, [27, 28]. One can treat it as an effective theory given by one-loop effective action that can be regularized and renormalized in some appropriate way. Of course, exact meaning of finite and divergent terms of effective action at $d>4$ can be understood only from the corresponding fundamental theory. In our article we consider the strong-field asymptotic behavior of the one-loop effective action. One can see that this asymptotic behavior is insensitive to methods of reg- ularization and renormalization, see, for example, [6, 32. At $d>4$ one can give a precise meaning and calculate the one-loop effective action using zeta-function regularization 31]. An application of this method to the case of a uniform magnetic field and self-dual field in arbitrary dimensions is described in detail in [32]. It can be shown that for $d \leq 4$ such a renormalization is equivalent to the above mentioned Schwinger's renormalization, see 32. Let us consider the application of this technique for the case of a constant uniform electric field in arbitrary dimensions of interest here.

First, we remind that the effective action $W$ from (49) can be represented as the functional determinant,

$$
W=-\frac{i}{2} \ln \operatorname{det} M^{2}, \quad M^{2}=m^{2}-i 0-P^{2}+\frac{q}{2} \sigma^{\mu \nu} F_{\mu \nu} .
$$

The operator $M^{2}$ becomes elliptic when it is continued to the Euclidean space, $M^{2} \rightarrow \tilde{M}^{2}$, by means of the replacements $t \rightarrow-i \eta, \partial_{0} \rightarrow i \partial_{\eta}$, and $q E \rightarrow i B$. Then the functional determinant is well-defined with the help of the zeta-function $\zeta^{(d)}(s)$ in $d$ dimensions,

$$
\begin{aligned}
& \ln \operatorname{det} \tilde{M}^{2}=-\left.\frac{d \zeta^{(d)}(s)}{d s}\right|_{s=0}, \\
& \zeta^{(d)}(s)=\Gamma^{-1}(s) \int_{0}^{\infty} d u u^{s-1} K(u),
\end{aligned}
$$

where $K(u)$ is the heat kernel,

$$
\begin{aligned}
& K(u)=\int d \eta d \mathbf{x} \operatorname{tr} f_{E u c l}(x, x, u) \\
& f_{E u c l}(x, x, u)=\left\langle\eta, \mathbf{x}\left|\exp \left(-u \tilde{M}^{2} / \mu^{2}\right)\right| \eta, \mathbf{x}\right\rangle
\end{aligned}
$$

Here the quantity $\mu$ is a renormalization scale, which is introduced to keep the zeta-function dimensionless. The dependence on $\mu$ of the functional determinant corresponds to a finite renormalization.

The effective action $W$ can be written as the following integral over the Euclidean space time volume: $W=-i \int \mathcal{L} d \eta d \mathbf{x}$. It is real when it is continued to the imaginary electric field $q E \rightarrow i B$, so that $\tilde{W}=\left.W\right|_{q E=i B}$, $\operatorname{Im} \tilde{W}=0$. Note that for $d \geq 3, \tilde{W}$ coincides with the effective action of the magnetic field $B / q$. Consequently, we obtain a regularized and finite form of the effective Lagrangian of a constant electric field in an arbitrary $d$ dimensions as follows:

$$
\operatorname{Re} \mathcal{L}_{r e g}=\left.\operatorname{Re} \tilde{\mathcal{L}}\right|_{B=-i q E}, \quad \tilde{\mathcal{L}}=-\left.\frac{1}{2} \frac{d \zeta^{(d)}(s)}{d s}\right|_{s=0} \Omega_{(d)}^{-1},
$$

where $\Omega_{(d)}=\int d \eta d \mathbf{x}$ is the Euclidean space-time volume in $d$ dimensions.

An explicit form of the quantity $\operatorname{tr} f_{E u c l}(x, x, u)$ can be extracted from the quantity $\operatorname{tr} f(x, x, s)$ (given by (39) and (48)) as follows:

$$
\operatorname{tr} f_{E u c l}(x, x, u)=-\left.\operatorname{tr} f\left(x, x,-\frac{i u}{\mu^{2}}\right)\right|_{q E=i B} .
$$


Then we have:

$$
\begin{aligned}
\zeta^{(2)}(s)= & \frac{\Omega_{(2)}}{2 \pi} \frac{1}{\Gamma(s)} \int_{0}^{\infty} u^{s-1} B \operatorname{coth}\left(\frac{B u}{\mu^{2}}\right) \mathrm{e}^{-m^{2} u / \mu^{2}} d u \\
\zeta^{(d)}(s)= & \frac{\Omega_{(d)} 2^{[d / 2]-1}}{\Omega_{(2)}}\left(\frac{\mu^{2}}{4 \pi}\right)^{d / 2-1} \frac{\Gamma(s-d / 2+1)}{\Gamma(s)} \\
& \times \zeta^{(2)}(s-d / 2+1), \quad \text { for } d>2 .
\end{aligned}
$$

If $B>0$, the function $\zeta^{(2)}(s)$ can be written in terms of Hurwitz zeta-function as follows [31, 32]:

$\zeta^{(2)}(s)=\left\{\begin{array}{rr}\frac{\Omega_{(2)} B}{2 \pi}\left[2\left(\frac{2 B}{\mu^{2}}\right)^{-s} \zeta_{H}\left(s, 1+\frac{m^{2}}{2 B}\right)+\left(\frac{m^{2}}{\mu^{2}}\right)^{-s}\right] & \text { for } m \neq 0 \\ \Omega_{(2)} \frac{B}{\pi}\left(\frac{2 B}{\mu^{2}}\right)^{-s} \zeta_{R}(s), & \text { for } m=0 .\end{array}\right.$

The Hurwitz zeta-function is defined as an analytic continuation of the series

$$
\zeta_{H}(s ; x)=\sum_{n=0}^{\infty}(n+x)^{-s}, \quad \operatorname{Re} s>1,
$$

to the entire complex plane of $s$. One can see that $\zeta_{R}(s)=\zeta_{H}(s, 1)$ is the ordinary Riemann zeta-function.
For odd $d$, and taking into account that $\Gamma^{-1}(s) \approx s$ at $s \approx 0$, we get from (56) that

$$
\left.\frac{d \zeta^{(d)}(s)}{d s}\right|_{s=0}=\left.\Gamma(s) \zeta^{(d)}(s)\right|_{s=0} .
$$

By using Eq. (54) we find a finite expression for $\operatorname{Re} \mathcal{L}_{\text {reg }}$ in arbitrary dimensions. Then we have to implement an additional finite renormalization of the cosmological constant and the electric charge [32]. For $d>4$, a finite renormalization of some high-dimensional quantities could be needed. Thus, we obtain the final form of the renormalized effective Lagrangian $\operatorname{Re} \mathcal{L}_{\text {ren }}$. The corresponding final forms for $\tilde{\mathcal{L}}$ and for the $B$ field in $d=2,3,4$ dimensions are treated in detail in 32 .

We are interested in the case of a very strong field, $m^{2} /|q E| \ll 1$. In this case the leading contribution to $\operatorname{Re} \mathcal{L}_{r e n}$ is given by $\operatorname{Re} \mathcal{L}_{\text {reg }}$ in (54), where $m \rightarrow 0$. Using (56), (57), and (58), we find that this contribution has the form

$\operatorname{Re} \mathcal{L}_{r e n} \approx \frac{1}{2} \operatorname{Re}\left\{\begin{array}{l}{\left[\ln \left(\frac{B}{\mu^{2}}\right) \frac{\zeta^{(d)}(0)}{\Omega_{(d)}}\right]_{B=-i q E}, \text { for even } d} \\ -\left[\left.\Gamma(s) \frac{\zeta^{(d)}(s)}{\Omega_{(d)}}\right|_{s=0}\right]_{B=-i q E}, \text { for odd } d\end{array}\right.$

In particular,

$$
\operatorname{Re} \mathcal{L}_{r e n} \approx \begin{cases}-\left.\operatorname{Re}\left[\frac{B}{4 \pi} \ln \left(\frac{B}{\mu^{2}}\right)\right]\right|_{B=-i q E}=\frac{|q E|}{4}, & \text { for } d=2, \\ -\left.\operatorname{Re}\left[\frac{1}{2 \pi^{2}}\left(\frac{B}{2}\right)^{3 / 2} \zeta_{R}\left(\frac{3}{2}\right)\right]\right|_{B=-i q E}=\frac{|q E|^{3 / 2}}{8 \pi^{2}} \zeta_{R}\left(\frac{3}{2}\right), & \text { for } d=3, \\ \left.\operatorname{Re}\left[\frac{B^{2}}{24 \pi^{2}} \ln \left(\frac{B}{\mu^{2}}\right)\right]\right|_{B=-i q E}=-\frac{(q E)^{2}}{24 \pi^{2}} \ln \left(\frac{|q E|}{\mu^{2}}\right), & \text { for } d=4 .\end{cases}
$$

For $d=4$, the obtained $\operatorname{Re} \mathcal{L}_{\text {ren }}$ coincides with the already known result [6]. For the magnetic field, the leading term in the form of the second Eq. (60) has previously been obtained in [28] for $d=3$. Note that despite the fact that the nonzero vacuum current $\left\langle j^{\mu}(t)\right\rangle^{c}$ is present in $d=3$ dimensions, the Chern-Simons term vanishes for the constant field strength [28]. In general, we have for a very strong electric field that

$$
\operatorname{Re} \mathcal{L}_{r e n} \sim \begin{cases}|q E|^{d / 2}, & d \neq 4 n \\ |q E|^{d / 2} \ln \left(|q E| / \mu^{2}\right), & d=4 n .\end{cases}
$$

In contrast to the electric field case, where the logarithmic factor $\ln \left(|q E| / \mu^{2}\right)$ appears only for $d=4 n$, in a strong magnetic field it is present in any even dimension: $\operatorname{Re} \mathcal{L}_{\text {ren }} \sim B^{d / 2} \ln \left(B / \mu^{2}\right)$ for even $d$, and $\operatorname{Re} \mathcal{L}_{\text {ren }} \sim B^{d / 2}$ for odd $d$. In the framework of the on-shell renormalization of massive theory, we have to set $\mu=m$.

Thus, we have obtained the renormalized mean values of EMT components in the following form:

$$
\begin{aligned}
\operatorname{Re}\left\langle T_{00}(t)\right\rangle_{\text {ren }}^{c} & =-\operatorname{Re}\left\langle T_{11}(t)\right\rangle_{\text {ren }}^{c} \\
& =E \frac{\partial \operatorname{Re} \mathcal{L}_{\text {ren }}(t)}{\partial E}-\operatorname{Re} \mathcal{L}_{\text {ren }}(t), \\
\operatorname{Re}\left\langle T_{i i}(t)\right\rangle_{\text {ren }}^{c} & =\operatorname{Re} \mathcal{L}_{\text {ren }}(t), \quad i=2,3, \ldots, D,
\end{aligned}
$$

where $\mathcal{L}(t)_{\text {ren }}$ at $t \in$ Int is given by (59). Thus, in the strong-field case, the quantities (61) have the following behavior:

$$
\operatorname{Re}\left\langle T_{\mu \mu}(t)\right\rangle_{r e n}^{c} \sim\left\{\begin{array}{l}
|q E|^{d / 2} \ln \left(|q E| / \mu^{2}\right), \quad d=4 n, \\
|q E|^{d / 2}, \quad d \neq 4 n, n \in \mathbb{N} .
\end{array}\right.
$$

At early $t<t_{\text {in }}$ and late $t>t_{\text {out }}$ times, we have $\operatorname{Re} \mathcal{L}(t)_{\text {ren }}=0$ and $\operatorname{Re}\left\langle T_{\mu \nu}(t)\right\rangle_{\text {ren }}^{c}=0$. Taking into account (61), we find the final form for the vacuum mean values of the EMT

$$
\left\langle T_{\mu \nu}(t)\right\rangle_{r e n}=\operatorname{Re}\left\langle T_{\mu \nu}(t)\right\rangle_{r e n}^{c}+\operatorname{Re}\left\langle T_{\mu \nu}(t)\right\rangle^{p},
$$


where, according to (46), the off-diagonal elements are equal to zero, and the diagonal elements of $\operatorname{Re}\left\langle T_{\mu \nu}(t)\right\rangle_{\text {ren }}^{c}$ are given by (61); the diagonal components of $\operatorname{Re}\left\langle T_{\mu \nu}(t)\right\rangle^{p}$ are studied in detail in the next section.

\section{E. Mean values: pair-creation contributions}

We know that the mean values $\left\langle j^{1}(t)\right\rangle^{p, \bar{p}}$ and $\left\langle T_{\mu \mu}(t)\right\rangle^{p, \bar{p}}$ are divergent as $T \rightarrow \infty$. Therefore, in the representation (35), they have to be considered always at finite $T$. Let us evaluate these quantities in the large $\tau$-limit approximation with $\tau=\sqrt{|q E|}\left(t-t_{i n}\right)$. Consider the time $t \in I n t$ for which the interval $t-t_{\text {in }}$ satisfies condition (37) and the cutoff approximations

$$
\begin{aligned}
& \left|\mathbf{p}_{\perp}\right| \leq \sqrt{|q E|}\left[\sqrt{|q E|}\left(t-t_{i n}\right)-K\right]^{1 / 2}, \\
& t_{i n}+K / \sqrt{|q E|} \leq p_{1} / q E \leq t-K / \sqrt{|q E|},
\end{aligned}
$$

hold true. In Eqs. (64) it is taken into account that physical observables in the time moment $t \in$ Int are affected by the electric field that acted for the time $\left[t_{i n}, t\right]$. Let us call the corresponding region in the momentum space by $\Omega(t)$. Using the transformation (2), one can represent the propagator $S^{p}$ in Eq. (29) in terms of out-solutions. In the asymptotic regime, as $z \rightarrow \infty$, the Weber functions have the following asymptotic expansion:

$$
D_{\nu}(z) \simeq z^{\nu} \exp \left(-z^{2} / 4\right)\left[\sum_{n=0}^{N} \frac{\left(-\frac{\nu}{2}\right)_{n}\left(\frac{1}{2}-\frac{\nu}{2}\right)_{n}}{n !\left(-z^{2} / 2\right)^{n}}\right],
$$

which is valid for $|\arg z|<3 \pi / 4$. Then, keeping only the zeroth $(n=0)$ term in last equation, we obtain for $x \simeq x^{\prime}$ :

$$
\begin{aligned}
S^{p}\left(x, x^{\prime}\right)=(\gamma P & +M) \Delta^{p}\left(x, x^{\prime}\right) \\
\Delta^{p}\left(x, x^{\prime}\right)=- & i \int d \mathbf{p} 2\left|q E t-p_{1}\right| \aleph_{\mathbf{p}} e^{i \mathbf{p} \cdot\left(\mathbf{x}-\mathbf{x}^{\prime}\right)} \\
\times & {\left[{ }^{+} \varphi_{\mathbf{p},-1}(t)^{+} \varphi_{\mathbf{p},-1}^{*}\left(t^{\prime}\right)\right.} \\
& \left.+{ }^{-} \varphi_{\mathbf{p},+1}(t)^{-} \varphi_{\mathbf{p},+1}^{*}\left(t^{\prime}\right)\right]
\end{aligned}
$$

Considering the large $\tau$-limit in representations (35), the domain of integration in (66) can be restricted to the region $\Omega(t)$ described by the inequalities (64). Using Eq. (14) for the differential mean values $\aleph_{p}$, we obtain:

$$
\begin{aligned}
\Delta^{p}\left(x, x^{\prime}\right)= & -i \int_{t_{i n}+K / \sqrt{|q E|}}^{t-K / \sqrt{|q E|}} h_{\perp}\left(\mathbf{x}_{\perp}, \mathbf{x}_{\perp}^{\prime}\right) h_{\|}\left(x_{\|}, x_{\|}^{\prime}\right) d \tilde{t}, \\
h_{\|}\left(x_{\|}, x_{\|}^{\prime}\right)= & \frac{1}{t-\tilde{t}} \mathrm{e}^{i p_{1}\left(x_{1}-x_{1}^{\prime}\right)} \cos \left\{\frac{1}{2}\left[\xi\left(t^{\prime}\right)^{2}-\xi(t)^{2}\right]\right\}, \\
h_{\perp}\left(x_{\perp}, x_{\perp}^{\prime}\right)= & \frac{|q E|^{d / 2-1}}{(2 \pi)^{d-1}} \\
& \times \exp \left(-\frac{\pi m^{2}}{|q E|}-\frac{\left(\mathbf{x}_{\perp}-\mathbf{x}_{\perp}^{\prime}\right)^{2}|q E|}{4 \pi}\right),
\end{aligned}
$$

where $p_{1}=q E \tilde{t}$.

It follows from Eqs. (35) and (67) that

$$
\begin{aligned}
& \left\langle j^{1}(t)\right\rangle^{p}=-\left.i q 2^{[d / 2]} P_{1} \Delta^{p}\left(x, x^{\prime}\right)\right|_{x=x^{\prime}}, \\
& \left\langle T_{\mu \mu}(t)\right\rangle^{p}=\left.i 2^{[d / 2]} P_{\mu}^{2} \Delta^{p}\left(x, x^{\prime}\right)\right|_{x=x^{\prime}} .
\end{aligned}
$$

Integrating over $p_{1}$, we obtain the following result in the large $\tau$-limit (with $\tau=\sqrt{|q E|}\left(t-t_{\text {in }}\right)$ ):

$$
\begin{aligned}
\left\langle j^{1}(t)\right\rangle^{p} & =2 e \operatorname{sgn}(E) r^{c r}\left[t-t_{i n}+|q E|^{-1 / 2} O(K)\right], \\
\left\langle T_{00}(t)\right\rangle^{p}= & \left\langle T_{11}(t)\right\rangle^{p} \\
= & |q E| r^{c r}\left[t-t_{i n}+|q E|^{-1 / 2} O(K)\right]^{2}, \\
\left\langle T_{i i}(t)\right\rangle^{p}= & \pi^{-1} r^{c r}\left\{\ln \left[\sqrt{|q E|}\left(t-t_{i n}\right)\right]+O(\ln K)\right\}, \\
& \text { for } i=2,3, \ldots, D,
\end{aligned}
$$

where $r^{c r}$ is given by (15). We see that all the leading contributions given by (69) and (70) are real. The quantities $\left\langle j^{1}(t)\right\rangle^{p}$ and $\left\langle T_{\mu \mu}(t)\right\rangle^{p}$ depend on the time interval $\left(t-t_{i n}\right)$ of the electric field action, showing that they are global quantities.

Now we estimate, for $t \approx t_{\text {out }}$, the current density and EMT of created particles, $j_{c r}^{\mu}(t), T_{\mu \nu}^{c r}(t)$, given by Eqs. (36). At this time instant, the solutions ${ }^{ \pm} \psi_{\mathbf{p}, r}(x)$ reduce to free particle plane waves in agreement with Eq. (9). Thus, taking into account representation (32), one can see that the quantities $\left\langle j^{1}(t)\right\rangle^{\bar{p}}$ and $\left\langle T_{\mu \mu}(t)\right\rangle^{\bar{p}}$ from (35) can be neglected in the large $\tau$-limit. Using Eqs. (69) and (70), we obtain:

$$
\begin{aligned}
& j_{c r}^{\mu}(t) \simeq \delta_{\mu, 1}\left\langle j^{1}(t)\right\rangle^{p}, \\
& T_{\mu \nu}^{c r}(t) \simeq \delta_{\mu, \nu}\left\langle T_{\mu \mu}(t)\right\rangle^{p}, \quad \text { at } t \approx t_{\text {out }},
\end{aligned}
$$

where Eqs. (41), (42), and (46) were taken into account. For $t>t_{\text {out }}$ (after the electric field is switched off), the quantities $j_{c r}^{\mu}(t)$ and $T_{\mu \nu}^{c r}(t)$ are constant and retain their values at $t_{\text {out }}$; for this reason, we always have to set $t-t_{\text {in }}$ $=T$ in such cases. The renormalized vacuum polarization contributions in expressions (43) and (63) vanish in the absence of external field. Therefore, for $t>t_{\text {out }}$, the vacuum mean values $\left\langle j^{\mu}(t)\right\rangle$ and $\left.T_{\mu \nu}(t)\right\rangle_{\text {ren }}$ represent the mean current density and EMT of pairs created by the complete $T$-constant electric field:

$$
\left\langle j^{\mu}(t)\right\rangle=j_{\text {cr }}^{\mu}\left(t_{\text {out }}\right), \quad\left\langle T_{\mu \nu}(t)\right\rangle_{\text {ren }}=T_{\mu \nu}^{c r}\left(t_{\text {out }}\right), \quad t>t_{\text {out }} .
$$

We see that the current density of created particles $j_{c r}^{\mu}(t)$ is directed along the direction of the electric field. In $d=3$ dimensions, in contrast to this almost obvious property, for $t_{\text {in }}<t<t_{\text {out }}$, the mean current density of each massive fermion specie deviates from the direction of the electric field. Indeed,

$$
\frac{\left\langle j^{2}(t)\right\rangle}{\left\langle j^{1}(t)\right\rangle}= \pm \frac{\sqrt{\pi}}{2} \gamma\left(\frac{1}{2}, \frac{\pi m^{2}}{|q E|}\right) \frac{\exp \left(\pi m^{2} /|q E|\right)}{\sqrt{|q E|}\left(t-t_{i n}\right)},
$$


where $(\sqrt{|q E|} T)^{-1} \ll 1$ according to stabilization condition (13). One finds a similar deviation of the mean current density of each massive fermion species in higher odd dimensions for $t_{\text {in }}<t<t_{\text {out }}$ in the case of an electric-like constant electromagnetic field, when all eigenvalues of the field tensor $F_{\mu \nu}$ are different from zero. The total mean current density of an even number of fermion species is directed along the direction of the electric field, since the contributions of the \pm -fermions differ only in sign.

One can see from Eq. (69) that $r^{c r} T$ is the total number density of pairs created and accelerated during the time $T$ to velocities nearly the speed of light. It coincides with the quantity $n^{c r}$ obtained in a different manner in (15). The quantity $T_{00}^{c r}=|q E| T n^{c r}$ is the mean energy density of pairs created at any time instant $t \in I n t$ with zero longitudinal kinetic momentum and then uniformly accelerated to kinetic momenta from zero to the maximum $|q E| T$, so that $|q E| T / 2$ is the mean kinetic momentum per particle. The energy density $T_{00}^{c r}$ is equal to the pressure $T_{11}^{c r}$ along the direction of the electric field. This equality is a natural equation of state for noninteracting particles accelerated by an electric field to relativistic velocities. The momentum density of created pairs $T_{0 i}^{c r}$ is zero due to the symmetry between particle and antiparticle distributions.

The vacuum mean values (43) and (63) for $t \in$ Int are sources in equations of motion for mean electromagnetic and metric fields respectively. It should be noted that only when the time $t$ is sufficiently close to $t_{\text {out }}$, $\left(t_{\text {out }}-t\right) / T \ll 1$, the differences between the densities $\left\langle j^{1}(t)\right\rangle^{p}$ and $\left\langle T_{\mu \nu}(t)\right\rangle^{p}$ and the respective densities $j_{c r}^{1}(t)$ and $T_{\mu \nu}^{c r}(t)$ of final pairs created, can be neglected and the interpretation of particles at $t$ as final out-particles is correct.

In the general case when the time $t$ is not close to $t_{\text {out }}$, there is an essential difference between the definition of the vacuum at $t<t_{\text {out }}$ and the final vacuum state $\mid 0$, out $\rangle$ at $t_{\text {out }}$. That is why the quantities $\left\langle j^{1}(t)\right\rangle^{p}$ and $\left\langle T_{\mu \nu}(t)\right\rangle^{p}$ have nothing to do with characteristics of final out-particles. They present contributions to mean values due to vacuum instability which depend on the history of the process, that is, they are global quantities, in contrast of the local quantities $\left\langle j^{\mu}(t)\right\rangle^{c},\left\langle j^{\mu}(t)\right\rangle_{\perp}^{p}$, and $\operatorname{Re}\left\langle T_{\mu \nu}(t)\right\rangle^{c}$. In the general case, $r^{c r}$ in Eqs. (69) and (70) is the total number density of excited states per unit of time. For example, the longitudinal component of the mean current density $\left\langle j^{1}(t)\right\rangle^{p}$ increases linearly as $\left(t-t_{\text {in }}\right)$ grows, since the decoherence does not take place for $t<t_{\text {out }}$. We note that $\left\langle j^{1}\left(t_{\text {out }}\right)\right\rangle \approx j_{\text {cr }}^{1}\left(t_{\text {out }}\right)$. However, it maybe not so if the decoherence starts earlier, for example, at the time instant $t_{d e c}, t_{d e c}<t_{\text {out }}$, and $t_{\text {out }}-t_{\text {dec }}$ is macroscopic; see discussion in the end of Section [II. In this case, the quantity $\left\langle j^{1}\left(t_{\text {out }}\right)\right\rangle$ decreases significantly in low-dimensional systems, and increases in high-dimensional systems.

We can compare contributions from the vacuum instability with contributions from the vacuum polarization.
Of course, all contributions due to pair creation in expressions (63) are exponentially small for the weak electric field, $m^{2} /|q E| \gg 1$, so that the vacuum polarization terms are principal. We are interested in the strong-field limit, $m^{2} /|q E| \ll 1$. In such a limit, we obtain from (70) that

$$
\begin{aligned}
& \left\langle T_{00}(t)\right\rangle^{p}=\left\langle T_{11}(t)\right\rangle^{p} \sim|q E|^{d / 2}|q E|\left(t-t_{i n}\right)^{2}, \\
& \left\langle T_{i i}(t)\right\rangle^{p} \sim|q E|^{d / 2} \ln \left[\sqrt{|q E|}\left(t-t_{i n}\right)\right], \quad i=2,3, \ldots, D,
\end{aligned}
$$

where the large dimensionless parameter $\sqrt{|q E|}\left(t-t_{i n}\right)$ satisfies the stabilization condition (37). Comparing the evaluation of the EMT components from (74) and (62), we see immediately that, when $d$ is not a multiple of four, the energy density of vacuum polarization, $\operatorname{Re}\left\langle T_{00}(t)\right\rangle_{\text {ren }}^{c}$, is negligible compared to the energy density due to pair creation, $\left\langle T_{00}(t)\right\rangle^{p}$, due to inequality (37).

If $d=4$, the ratio $\left|\left\langle T_{00}(t)\right\rangle^{p} / \operatorname{Re}\left\langle T_{00}(t)\right\rangle^{c}\right|$ in a massive theory with on-shell renormalization, $\mu=m$, is of the order $|q E|\left(t-t_{i n}\right)^{2} / \ln \left(|q E| / m^{2}\right)$. In order to estimate the allowed values of the logarithm in the latter equation, we have to have a physical model that describes the origin of the external classical quasiconstant electric field. In problems of high-energy physics it is usually assumed that just from the beginning there exists an uniform classical electric field having a given energy density. The system of fermions interacting with this field is closed, that is, the total energy of the system is conserved. Under such an assumption, we take into account that quantum electrodynamics in $d=4$ dimensions with the strong $T$-constant external electric field can be considered as a consistent model only if the backreaction due to pair creation is relatively small with respect to the background, which implies the following restriction from above:

$$
|q E|\left(t-t_{i n}\right)^{2} \ll \frac{\pi^{2}}{2 \alpha},
$$

where $\alpha$ is the fine structure constant, see 10 , 33]. We consider macroscopic time intervals, such that $m^{2}\left(t-t_{i n}\right)^{2} \gg 1$, then it follows from (75) that $|q E| / m^{2} \ll \pi^{2} /(2 \alpha)$. Whence we obtain $\ln \left(|q E| / m^{2}\right) \ll 6.5$. On the other hand, according to condition (75), the maximum value allowed for $|q E|\left(t-t_{i n}\right)^{2}$ is two orders of magnitude greater than the restriction obtained for the logarithm. Thus, we see that in this case the quantity $\operatorname{Re}\left\langle T_{00}(t)\right\rangle_{\text {ren }}^{c}$ is negligible in comparison with $\left\langle T_{00}(t)\right\rangle^{p}$. This result can be generalized to $d=4 n$ dimensions. Note that the ratio of the transverse components of the pressure $\left|\left\langle T_{i i}(t)\right\rangle^{p} / \operatorname{Re}\left\langle T_{i i}(t)\right\rangle^{c}\right|$ is of the order $\ln \left[\sqrt{|q E|}\left(t-t_{i n}\right)\right]$ if $d \neq 4 n$ and $\ln \left[\sqrt{|q E|}\left(t-t_{i n}\right)\right] / \ln \left(|q E| / m^{2}\right)$ if $d=$ $4 n$. One can see, for example, in $d=4$ dimensions, that these logarithms cannot be considered, in general, as really big quantities due to restriction from above (75) and 
this evaluation can be generalized to arbitrary dimensions. Then, in general, none of these transverse components can be neglected. The evaluations may be different when another model for the external classical quasiconstant field is considered, for example, when there is an external source that supports a given external field strength.

\section{MEAN CURRENT AND EMT IN GRAPHENE}

\section{A. One-loop results in a given external field}

It is known that at certain conditions electronic excitations in graphene monolayer (just graphene, in what follows) behave as relativistic Dirac massless fermions in $2+1$ dimensions. The so-called Dirac model for electronic excitations in graphene was developed first by Semenoff in 14], exploring results obtained decades earlier in the study of the conductivity of graphite [34], see details in recent reviews [12, 13]. It was found that, at zero temperature and chemical potential (i.e., at the socalled charge neutrality point), low-energy electronic excitations in graphene are described in a tight-binding approximation by the Dirac equation for massless particle in $2+1$ dimensions, with the Fermi velocity $v_{F} \simeq 10^{6} \mathrm{~m} / \mathrm{s}$ playing the role of the speed of light in relativistic particle dynamics. In this section, we are going to explore such a correspondence, and consider applications of the results obtained in the study of the quantized Dirac field in an external background presented in the previous sections to some problems of graphene physics that can be studied within the Dirac model. In fact, we are going to study the electronic transport in graphene at low carrier density and low temperatures when quantum interference effects are important. Accordingly, we shall restrict from now on to the massless case in $2+1$ dimensions.

First some comments about the Dirac model of graphene. There are actually two species of fermions in this model, corresponding to excitations about the two distinct Dirac points in the Brillouin zone of graphene, i.e., each species belongs to a distinct valley. The algebra of $\gamma$-matrices has two inequivalent representations in $(2+1)$-dimensions, as described in (40), and a distinct (pseudo spin) representation is associated with each Dirac point. There is no parity anomaly in the Dirac model, in particular, the sum of current densities $\left\langle j^{\mu}(t)\right\rangle^{c}$ (vacuum current contributions to the probability amplitudes for processes with photons) for the two fermion species, given by Eq. (41), is zero. Note that the mean value of transverse vacuum polarization current, given by Eq. (44), is equal to zero for each massless fermion species. For all other integral quantities, since intervalley scattering can be neglected, the presence of two valleys is taken into account simply by multiplying by the degeneracy factor 2 .

Furthermore, there also is a spin degeneracy factor.
The derivation of the Dirac model starts from a nonrelativistic Schrödinger equation for the conduction electrons in graphene, leading to another doubling of fields due to the (real) spin of the electron. As a result, there are four species of fermions in the Dirac model corresponding to graphene. The mean values that we have obtained in the previous section hold true for each of the Dirac fields independently. In order to find the corresponding mean values in graphene, one should first add the contributions from the two valleys, as discussed in the previous paragraph, and after that multiply by the spin degeneracy factor two.

We consider an infinite flat graphene sample on which a uniform electric field is applied, directed along the plane of the sample. We assume that the applied field is the $T$-constant electric field studied in the previous sections: the field is suddenly switched on at some time $t_{i n}$, acting then for a time-interval $T$, during which electron-hole pairs are created. We consider the case of zero temperature and chemical potential, so that the Dirac model can be used, and an initial state with neither electrons nor holes.

Under these circumstances, the Eqs. (15), (43), and (63), multiplied by a degeneracy factor of four, describe, respectively: $n_{g}^{c r}$, the total number density of electronhole pairs created by the electric field; $\left\langle j^{1}(t)\right\rangle_{g}$, the mean longitudinal current density; and $\left\langle T_{\mu \mu}(t)\right\rangle_{g}$, the mean EMT in the graphene. For the sake of comparison with known experimental results, we are going to use SI units and restore the Planck constant $\hbar$ in this part of the work. We get the following results:

$$
\begin{aligned}
& n_{g}^{c r}=r_{g}^{c r} T, \quad r_{g}^{c r}=\pi^{-2}\left(v_{F} \hbar^{3}\right)^{-1 / 2}|e E|^{3 / 2} ; \\
& \left\langle j^{1}(t)\right\rangle_{g}=\operatorname{sgn}(E) A \Delta t, \quad A=2 e v_{F} r_{g}^{c r} \\
& \left\langle T_{00}(t)\right\rangle_{g}=\left\langle T_{11}(t)\right\rangle_{g}=e|E| v_{F} r_{g}^{c r}(\Delta t)^{2} \\
& \left\langle T_{22}(t)\right\rangle_{g}=\left\langle T_{22}(t)\right\rangle_{g}^{p}+\left\langle T_{22}(t)\right\rangle_{g}^{c} \\
& \left\langle T_{22}(t)\right\rangle_{g}^{c}=\hbar r_{g}^{c r} \zeta_{R}(3 / 2) / 2 \\
& \left\langle T_{22}(t)\right\rangle_{g}^{p}=\hbar r_{g}^{c r} \pi^{-1} \ln \left(\sqrt{e|E| v_{F} / \hbar} \Delta t\right)
\end{aligned}
$$

where $\Delta t=t-t_{i n}$. These results hold true for all $t$ that satisfy the stabilization condition (37), which has now the form:

$$
\sqrt{e|E| v_{F} / \hbar} \Delta t \gg 1
$$

There appears a time scale specific to graphene,

$$
\Delta t_{s t}=\left(e|E| v_{F} / \hbar\right)^{-1 / 2} \text {, }
$$

which plays the role of the stabilization time in the case under consideration. The vacuum polarization contribution to the mean value $\left\langle T_{00}(t)\right\rangle_{g}=\left\langle T_{11}(t)\right\rangle_{g}$ in (78) is small due to Eq. (79) and is neglected, that is, $\left\langle T_{00}(t)\right\rangle_{g}=\left\langle T_{00}(t)\right\rangle_{g}^{p}$ and $\left\langle T_{11}(t)\right\rangle_{g}=\left\langle T_{11}(t)\right\rangle_{g}^{p}$.

At $t \approx t_{\text {out }}$, we have $\Delta t \approx T=t_{\text {out }}-t_{\text {in }}$ and relations (71) show that the mean values $\left\langle j^{1}(t)\right\rangle_{g}$ and $\left\langle T_{\mu \mu}(t)\right\rangle_{g}^{p}$ 
hardly differ from the current density $j_{c r}^{1}\left(t_{\text {out }}\right)$ and the quantity $T_{\mu \mu}^{c r}\left(t_{\text {out }}\right)$ caused by created particles. In the general case, the quantity $r_{g}^{c r}$ is the number density of pairs of positive and negative charged states excited due to the constant electric field per unit of time. Only at $t \approx t_{\text {out }}$, it can be treated as the production rate of electron-hole pairs. In the presence of a mass gap $\Delta \varepsilon=m v_{F}^{2}$, the rate $r_{g}^{c r}$ is attenuated by a factor of $\exp \left[-\pi(\Delta \varepsilon)^{2} / e|E| v_{F} \hbar\right]$ according to Eq. (15). In this case, the stabilization condition has general form (37) and the strong field condition reads $(\Delta \varepsilon)^{2} / e|E| v_{F} \hbar \ll 1$.

There is a huge amount of papers on the conductivity in graphene, for the most part on optical conductivity and on the minimal dc conductivity, see, for example, a recent review of electronic transport in graphene [13] and an analysis of the situation with the minimal dc conductivity in [16, 35]. It is shown in [16, 17] that the time scale $\Delta t_{s t}$ appears for the tight-binding model as the time scale when the perturbation theory with respect to electric field breaks down $\left(\Delta t_{s t} \gg t_{\gamma}\right.$, where the microscopic time scale is $t_{\gamma}=\hbar / \gamma \simeq 0.24 \mathrm{fs}$, with $\gamma=2.7 \mathrm{eV}$ being the hopping energy), and the dc response changes from the linear in $E$ time-independent regime to a non-linear in $E$ and time-dependent regime. Thus, it was established that the minimal dc conductivity occurs for ballistic flight times $\Delta t$ smaller than $\Delta t_{s t}$. Our expression (77) is obtained for the large time interval $\Delta t$ satisfying the condition (79). Now let us compare the results we have obtained with the known results for sufficiently large duration of electric field in the form of expression vs expression.

The formula for the current density of created particles $j_{c r}^{1}\left(t_{\text {out }}\right)$ that follows from expression (77) agrees with the result obtained from the WKB approach, see Eq. (8) in [4], Eqs. (20) and (25) in [15], and Eq.(A3) in [19] (the numerical analysis of [19] performed in the frame of a non-equilibrium Green function (NEGF) approach, which is referred to as a non-perturbative quantum mechanical approach, is consistent with the semiclassical result). In condensed-matter physics such a method is known as the Landau-Zener approach (Note that the WKB approach is valid when the differential mean number $\aleph_{\mathbf{p}}$, given by general expression (11), is small. However, the WKB approximation for $\aleph_{\mathbf{p}}$ coincides with the exact expression (14) in the limit $T \rightarrow \infty$ ). The time-dependence of mean current density $\left\langle j^{1}(t)\right\rangle_{g}$ given by Eq. (77) is consistent with the numerical solution of the first-quantized tight-binding model equations obtained for the ballistic case in the time interval $\Delta t_{s t}<\Delta t<\Delta t_{B}$, see Eq. (82) in Ref. [17]; there a factor $3^{3 / 4} 2^{-7 / 2}$ is replacing our $2 / \pi^{2}$, but these factors are both equal to 0.20 , numerically. The $\Delta t_{B}=2 \pi \hbar(e|E| a)^{-1}$ is the Bloch time, required for the constant electric field to shift the kinetic momentum across the Brillouin zone ( $a \approx 0.142 \mathrm{~nm}$ is the carbon-carbon distance) and, of course, this value falls beyond to what may be covered by the continuous Dirac model.

The expression for $\left\langle j^{1}(t)\right\rangle_{g}$ given by (77) is a key for- mula in the study of the conductivity in the graphene at low carrier density beyond the linear response in dc. It describes the mean electric current of coherent carriers produced by the applied electric field. An exotic feature of the electronic transport at the charge neutrality point, as described by the Dirac model, is that one begins without any charges to be accelerated at all: there are no electrons or holes initially. The induced current can be considered, if one likes semiclassical style comments, as a consequence of two mechanisms: charged pairs of ultrarelativistic (with the constant velocity $v_{F}$ ) coherent electrons and holes are first created with a strong suppression of large transverse momenta $\left(\left|p_{2}\right|>\sqrt{e|E| \hbar / v_{F}}\right)$ and after that their longitudinal kinetic momenta are coherently increased by the electric field. The combined effect of both processes that, in fact, cannot be localized and separated in the framework of QED, is described by Eqs. (77) and (78). In contrast to this, at high carrier density one has to consider the increment of the longitudinal kinetic momenta of an initially given number of incoherent carriers by the electric field. In the latter case the transport theory allows the semiclassical description of carriers.

It follows from our results that the mean current in the graphene is parallel to the applied field, proportional to $|E|^{3 / 2}$, and grows linearly with time. The fact that the current grows indefinitely as $T \rightarrow \infty$ is a consequence of the absence of scattering and a backreaction mechanisms in the model of unlimited size under consideration: only effects caused by the applied external field have been taken into account. In the experimental situation described in Ref. [19], a constant voltage between two electrodes connected to the graphene was applied, and current-voltage characteristics $(I-V)$ are measured within $\sim 1 \mathrm{~s}$, which is a very large time-scale comparing with the ballistic flight time $T_{b a l}$ (the time which the electron spends to cross the material of a finite length $\left.L_{x}\right), T_{b a l}=L_{x} / v_{F}$. To match our results with these conditions, our time $T$ should be replaced by some typical time-scale that we call the effective time duration $T_{\text {eff }}$. Some kind of dissipation process may truncate the pair creation at $T_{d i s}$, in which case $T_{\text {eff }}=T_{d i s}$. The standard candidates are collisions with impurities, phonons, ripplons, and the electron-electron interactions. A description of how the pair creation is counterbalanced by dissipative processes so that a stable current settles down is still an open question. In the absence of the dissipation, the transport is ballistic; in this case, considering a strip with lateral infinite width and a finite length $L_{x}$, we assume the ballistic flight time $T_{b a l}$ to be the effective time duration, $T_{\text {eff }}=T_{b a l}$. It is experimentally shown, see [13, 36] and references therein, that the low temperature $(\Theta \sim 5 \mathrm{~K})$ and gate voltage $\left(\left|V_{g}\right|<5 \mathrm{~V}\right)$ transport in current-annealed, suspended devices with a mobility $\sim 170000 \mathrm{~cm}^{2} / \mathrm{Vs}$ is close to the ballistic limit over micron dimensions. In a realistic sample, placed on a substrate, the effective time duration $T_{\text {eff }}$ can be many 
times smaller than $T_{b a l}$, because of charged impurities or structural disorder of the substrate. However, such an effective time $T_{\text {eff }}$ remains macroscopically large, so that Eq. (79) still holds. One can expect that in realistic high-quality (high mobility) samples the time $T_{\text {eff }}$ is comparable with the time $T_{b a l}$. In any case, for a finite flake length, the potential difference $V=E L_{x}$ is finite, and one can consider the $I-V$ of graphene devices. Then Eq. (77) describes a regime where the current behaves as $j \sim V^{3 / 2}$.

This fractional power dependence of the currentvoltage characteristic in graphene is at present called nonlinear (or superlinear) transport in graphene $(I-V$ of the form $\left.j \sim V^{3 / 2}\right)$. In the Ref. [4, 15, 17], this behavior was related to a possible pair creation by a constant electric field and experiments aiming at the observation of the effect were proposed. An experimental observation was recently reported [19]. It is agreed that such a superlinear transport is a distinctive feature characterizing the regime dominated by pair creation.

It should be noted that our description of the quantum transport in graphene in the framework of strong-field QED is not restricted by a semiclassical approximation of carriers and it does not use any statistical assumptions inherent in the Boltzmann transport theory. The estimations that follow show that this is important for the study of the conductivity close to the Dirac point. A typical density $n_{q}^{c r}$ of carriers created in the ballistic case, given by Eq. (76), e.g. for typical $V \sim 1 \mathrm{~V}$ and $L_{x} \sim 1$ $\mu \mathrm{m}$, is of the order $n_{g}^{c r} \sim 6 \times 10^{11} \mathrm{~cm}^{-2}$. Thus, in the general case, the density $n_{g}^{c r}$ is of the same order as the impurity density $n_{i m p}\left(10^{10}-10^{12} \mathrm{~cm}^{-2}[13]\right)$. It should be stressed that this estimate is made for the ballistic case when $T_{e f f}=T_{b a l}$. If $T_{e f f} \ll T_{b a l}$, then the density $n_{g}^{c r}$ is less than $n_{i m p}$. Moreover, the electric field creates the carriers in pure states with distribution (14) that differs significantly from the equilibrium distribution. The carriers created at relatively small times $T_{\text {eff }} \sim \Delta t_{s t}$ are wave packets with partial plane waves, which have wide range of wave lengths from $\infty$ to approximately $0.2 \mu \mathrm{m}$. These lengths correspond to maximum kinetic momenta of the order $\sqrt{e|E| \hbar / v_{F}}$, see (14). As $T_{\text {eff }}$ increases the range of the longitudinal kinetic momenta of created particle grows to its maximum $e|E| T_{\text {eff }} \gg \sqrt{e|E| \hbar / v_{F}}$; at the same time the range of their transverse kinetic momenta remains unchanged. Thus, in the ballistic case, the lower bound of the range of the longitudinal wavelengths decreases achieving its minimum of the order 4 $\mathrm{nm}$. The estimated minimum wavelength of the order $0.2 \mu \mathrm{m}$ is not much less than sample sizes that were used in experiments, and in some cases they are of the same order. Thus, we see that the Boltzmann theory, based on WKB approximation, is not well adapted to describe evolution of massless carries created from the vacuum. This is a nonstandard situation for usual transport problems in condensed matter physics. Our approach gives a nonperturbative description of the system evolution when quantum interference effects are important. We see that the QED derivation of the superlinear $I-V$ presented above gives additional arguments in favor of the interpretation that such a behavior is due to the pair creation. At the same time, in order to continue the study of the conductivity of graphene in the framework of the QED methods, one has to analyze in detail physical conditions in the graphene under which all the machinery, and especially Eqs. (77) and (78), are valid.

\section{B. Mean electromagnetic field}

The mean time-dependent current $\left\langle j^{1}(t)\right\rangle_{g}$ is the source in the Maxwell equations for a mean electromagnetic field $(\overline{\mathbf{E}}, \overline{\mathbf{B}})$, where $\overline{\mathbf{E}}=\mathbf{E}+\mathbf{E}_{r a d}$ and $\overline{\mathbf{B}}=\mathbf{B}_{r a d}$ are electric and magnetic components, respectively. Here, the initial external constant uniform electric field $\mathbf{E}$ satisfies the homogeneous Maxwell equations, and the fields $\mathbf{E}_{r a d}$ and $\mathbf{B}_{\text {rad }}$ are due to the current $\left\langle j^{1}(t)\right\rangle_{g}$, representing the backreaction of created pairs to the external field. The charged fermions in the graphene should feel the total field $(\overline{\mathbf{E}}, \overline{\mathbf{B}})$; in particular, the pair creation is induced by the mean electric field $\overline{\mathbf{E}}$. Since the current $\left\langle j^{1}(t)\right\rangle_{g}$ increases linearly as $\Delta t$ increases, the backreaction to the external field should become relevant at some time instant. The situation looks similar to that studied in [10, 33] for QED in 3+1 dimensions, although there are considerable peculiarities in the case under consideration. Dealing with graphene devices, it is natural to assume that the constant strength $E$ on the graphene plane is due to the applied fixed voltage $V$ and therefore is not changed when the created charges flow into the reservoirs which are located outside the graphene and have sufficiently large capacitances. It is assumed that an external current flows to the electrodes to maintain the fixed voltage, i.e., we are dealing with an open system of fermions interacting with classical electromagnetic field. Then Maxwell equations can be solved through infinite space and we may assume that the strength $E$ is fixed by boundary condition at the infinity. The electromagnetic field is not confined to the graphene surface, $z=0$, but rather propagates in the ambient $3+1$ dimensional space-time, where $z$ is the coordinate of axis normal to the graphene plane. To take this into account, we have to present the current term in the Maxwell equations as the current $\mathbf{J}=(J, 0,0)$ restricted to a plane immersed in the 3 -dimensional space. We are interested in the time intervals $\Delta t$ that satisfy the inequality $0 \leq \Delta t \leq T$. Then, taking into account that the current $\left\langle\bar{j}^{1}(t)\right\rangle_{g}$ appears for $\Delta t>0$, we can write $J=\left\langle j^{1}(t)\right\rangle_{g} \delta(z) \theta(\Delta t)$ for $\Delta t \leq T$. The nontrivial Maxwell equations to be solved are:

$$
\boldsymbol{\nabla} \times \overline{\mathbf{B}}=\mu_{0} \mathbf{J}+\frac{1}{c^{2}} \frac{\partial \overline{\mathbf{E}}}{\partial t}, \quad \boldsymbol{\nabla} \times \overline{\mathbf{E}}=-\frac{\partial \overline{\mathbf{B}}}{\partial t},
$$

where $\mu_{0}$ is the magnetic permeability and $c$ is the speed of light in the vacuum.

The only nonzero components of the irradiated field are the $x$-component of the electric field, $E_{x}^{r a d}$, and the 
$y$-component of the magnetic field, $B_{y}^{\text {rad }}$. Solving the Maxwell equations under initial condition $E_{x}^{r a d}=0$ and $B_{y}^{r a d}=0$ at $\Delta t=0$, one finds that for $0 \leq \Delta t \leq T$, the electromagnetic field produced by the current confined to the graphene sheet is:

$$
\begin{aligned}
& E_{x}^{r a d}=-\frac{\mu_{0}}{2} \operatorname{sgn}(E) A(c \Delta t-|z|) \theta(c \Delta t-|z|), \\
& B_{y}^{r a d}=\operatorname{sgn}(z) E_{x}^{r a d} / c .
\end{aligned}
$$

The direction of the induced magnetic field changes sign across the graphene sheet, and $\operatorname{sgn}(z)= \pm 1$ corresponds to upper and lower regions. The electric field $E_{x}^{r a d}$ is opposite to the applied field $E$ and is continuous in $z$-direction, in particular, at $z=0$. A real graphene flake is a film of very large dimension in the $x-y$ plane and a finite monolayer atomic thickness of approximately $0.1 \mathrm{~nm}$ to $0.2 \mathrm{~nm}$ in the $z$-direction [13]. For realistic densities of carriers the potential field in $z$-direction looks like a deep potential well, which effectively forbids the motion of carries in this direction. Then it is natural to assume that the induced electromagnetic field inside the graphene is the mean between limiting values of the field from upper and lower regions as $|z| \rightarrow 0$. Thus, the intensity of the induced electric field on the graphene plane reads:

$$
E_{x}^{r a d}=-\operatorname{sgn}(E) \frac{4 \alpha}{\pi}|E|^{3 / 2} \sqrt{v_{F} e / \hbar} \Delta t,
$$

where $\alpha=\mu_{0} c e^{2} / 2 h$ is the fine structure constant. As to the induced magnetic field, we believe that it is zero inside the graphene.

The QED with an external constant electric field is a consistent model as long as the field produced by the induced current is negligible compared to the applied field, $\left|E_{x}^{r a d}\right| \ll|E|$. This gives the consistency restriction:

$$
\sqrt{v_{F} e|E| / \hbar} \Delta t \ll \frac{\pi}{4 \alpha} .
$$

In this case, the external electric field can be considered as a good approximation of the effective mean field. We call the typical time scale related to Eq. (84), $\Delta t_{b r}=$ $\Delta t_{s t} \pi / 4 \alpha$, the time of backreaction. On the other hand, the dimensionless parameter in the l.h.s. of Eq. (84) satisfies the stabilization condition given by Eq. (79). Thus, there is a window in the parameter range where the model is consistent, $t_{\gamma} \ll \Delta t_{s t} \ll \Delta t_{b r}$. Moreover, this restriction corresponds to a specific regime which might be relevant to some known experiments with graphene, as we shall see below.

In the description of the carrier creation inside a graphene flake of a finite length $L_{x}$, we use a simple picture of the pair creation due to the $T$-constant field. We assume that the maximal duration of the electric field in our model is the effective time duration $T_{e f f}$, and that, in the case of ballistic transport, $T_{\text {eff }}=T_{b a l}$. In typical experiments, $L_{x} \sim 1 \mu \mathrm{m}$, and then $T_{b a l} \sim 10^{-12}$ s. Taking
$\Delta t=T_{b a l}$ in Eqs. (79) and (84), we obtain the following restrictions on the electric field:

$$
7 \times 10^{2} \mathrm{~V} / \mathrm{m} \ll|E| \ll 8 \times 10^{6} \mathrm{~V} / \mathrm{m} .
$$

Since the voltage is $V=E L_{x}$, one finds the inequalities

$$
7 \times 10^{-4} \mathrm{~V} \ll V \ll 8 \mathrm{~V} .
$$

These voltages are in the range typically used in experiments with graphene.

In the experiment described in [19], for instance, the $I-V$ curve was studied in a range from decivolts to a few volts, in samples with lengths $L_{x}$ varying from 0.9 to 5.9 $\mu \mathrm{m}$ and widths from 70 to $1500 \mathrm{~nm}$. A power law $I \sim V^{\delta}$ was used to fit the $I-V$ curves near the Dirac point, and it was found that $1 \leq \delta \leq 3 / 2$. It was shown that the $I-V^{\prime}$ s in the graphene devices become superlinear in the presence of disorder (in low mobility samples) while in high-quality (high mobility) samples, the superlinearity is masked by some other effect. The exponent $\delta$ looks like a monotonically decreasing function of mobility for different devices and superlinearity vanishes for devices with high mobility, $\delta \rightarrow 1$. These results were interpreted as an interplay between pair creation and scattering by charged impurities and optical phonons. According to this interpretation, the superlinearity of the $I-V$ in low mobility samples is in a qualitative agreement with expression (77), while it is compensated by the contribution of the intraband current (the current of carriers that were present before the electric field was switched on, due to an imperfect experimental realization of the Dirac point conditions), which tends to saturate in high mobility samples due to interaction with optical phonons. The absence of a compensation of the superlinearity in low mobility samples is explained by the fact that the presence of a large number of charged impurities prevents the growth of the intraband current, making the interaction with optical phonons irrelevant. Except for this qualitative analysis, however, there is no theoretical description of these observations; the numerical analysis (in the NEGF approach) of [19], in particular, does not include the optical phonons which are central to their interpretation of the effect, and it is argued that other effects should also be relevant for the analysis, as selfheating of the graphene sample, for instance [19].

In fact, according to our analysis, backreaction should not be neglected. We have shown that the consistency of the Dirac model with a given external field is ensured under the conditions (79) and (84) for $\Delta t$ and $E$, which are thus extremely important for obtaining adequate physical results in course of the calculations. Analyzing the experimental settings one can be sure that the stabilization condition (79) is satisfied for all known measurements, which means that the lower bound in (86) is respected. However, the condition (84) could be violated because the voltage varies from 1 to $5 \mathrm{~V}$, and sample length varies from 0.9 to $5.9 \mu \mathrm{m}$, as reported in [19]. Therefore, backreaction (appearance of the induced electric field $E_{x}^{r a d}$ ) cannot be ignored in calculating the mean 
current in high mobility samples. This means that, in the ballistic case, Eq. (77) can give overestimated values for the current density of created particles for a given voltage. In the case of low mobility samples, the effective duration time $T_{\text {eff }}$ is due to the scale of dissipation processes $T_{d i s}$, which can be many times less than the time $T_{b a l}$, so that condition (84) holds true at $\Delta t=T_{d i s}$, and the regime of backreaction is not reached. As mobility is increased, backreaction becomes more pronounced, and it might compensate the superlinearity of the pair-creation process. In order to investigate whether this effect can lead to a transition from a superlinear to a linear $I-V$ curve, we investigate in the next section how backreaction affects pair-production in graphene.

\section{Effective mean field and mean current}

In this section, we study a possible generalization of the above considered model with the $T$-constant external field. In particular, in the framework of this generalization we are going to take into account the backreaction of the mean current to the applied electric field.

Let us consider time intervals when the external electric field is switched on, $0 \leq \Delta t \leq T$. In realistic cases, after switching on, the external field $E$ remains constant on the graphene plane due to the applied voltage $V$, which is supported by external sources to remain fixed. Accordingly, we assume that on the graphene plane $E$ cannot be changed due to pair creation inside of the sample. As to the electric field $\overline{\mathbf{E}}$ inside the graphene, we suppose that it can vary with time and it is directed along the axis $x$, so that $\overline{\mathbf{E}}(t)=(\bar{E}(t), 0,0)$. The $x$-component $\bar{E}(t)$ is a superposition of the applied external field $E$ and a time-dependent electric field $E_{x}^{\text {rad }}(t)$ irradiated by the current induced in the sample, $\bar{E}(t)=E+E_{x}^{\text {rad }}(t)$. In the initial time instant $t_{1}=t_{\text {in }}$, we have $\bar{E}\left(t_{1}\right)=E$. Let $\bar{E}(t)$ be a slowly varying field within a time interval $\Delta t_{i}=t_{i+1}-t_{i}>0$ such that the following condition holds:

$$
\left|\frac{1}{\bar{E}(t)} \frac{\partial \bar{E}(t)}{\partial t}\right| \Delta t_{i} \ll 1, \quad t \in\left(t_{i}, t_{i+1}\right],
$$

and let $\Delta t_{i}$ be large enough to obey the stabilization condition of the type (79),

$$
\sqrt{e|\bar{E}(t)| v_{F} / \hbar} \Delta t_{i} \gg 1, \quad t \in\left(t_{i}, t_{i+1}\right] .
$$

We consider now the time evolution of the mean field and the vacuum mean current between the initial time $t_{\text {in }}$ when the external field is switched on and a time instant $t_{\text {fin }}$, which coincides with $t_{\text {out }}$, or precedes it, $t_{\text {fin }} \leq t_{\text {out }}$. We first divide the interval $\left(t_{\text {in }}, t_{\text {fin }}\right]$ into $N$ equal intervals $\Delta t_{i}$, such that $\Delta t_{1}=\Delta t_{2}=\cdots=\Delta t_{N}$, $\sum_{i=1}^{N} \Delta t_{i}=t_{f i n}-t_{i n}$. We suppose that Eqs. (87) and (88) hold true for all intervals. That allows us to treat the electric field as approximately constant within each interval, $\bar{E}(t) \approx \bar{E}\left(t_{i}\right)$, for $t \in\left(t_{i}, t_{i+1}\right]$.
Let us find the vacuum mean current density for the slowly varying electric field $\bar{E}(t)$. We begin with the case $t \in\left(t_{1}, t_{2}\right]$. Here, according to Eqs. (67) and (68), the current density $\left\langle j^{1}(t)\right\rangle_{g}$ is formed by the contributions from the vacuum states excited by the field $E$, these contributions having momenta that are restricted to the region (64). The phase volume of this region depends both on the magnitude of the electric field $E$ and on the interval $t-t_{1}$. It follows from the Eq. (67) that the density $\left\langle j^{1}(t)\right\rangle_{g}$ grows as $t-t_{1}$ because the field $E$ excites additional states with larger longitudinal momentum $p_{1}$. In the end of this time interval, when $t=t_{2}$, the current density $\left\langle j^{1}(t)\right\rangle_{g}$ takes the form:

$$
\Delta j_{1}=\operatorname{sgn}(E) D\left|\bar{E}\left(t_{1}\right)\right|^{3 / 2} \Delta t_{1},
$$

with $D=2 \pi^{-2} v_{F}^{1 / 2} \hbar^{-3 / 2} e^{5 / 2}$. According to Eq. (83), the field $E_{x}^{r a d}(t)$ has a direction opposite to $\bar{E}\left(\bar{t}_{1}\right)=E$, and from inequality (87) its magnitude is small in comparison with it, $\left|E_{x}^{r a d}(t)\right| \ll\left|\bar{E}\left(\bar{t}_{1}\right)\right|$. Therefore, Eq. (89) determines the leading term in the mean value $\left\langle j^{1}(t)\right\rangle_{g}$ in the corresponding large $\tau$-limit and in the mean field approximation, and any corrections to $\left\langle j^{1}(t)\right\rangle_{g}$ due to derivative $\partial_{t} \bar{E}(t)$ are small. One can see that $\left|\bar{E}\left(t_{2}\right)\right|<\left|\bar{E}\left(t_{1}\right)\right|$. Now let us proceed to the case $t \in\left(t_{2}, t_{3}\right]$. The effective mean field $\bar{E}(t)$ is already different from $E$ and, as before, the field $\bar{E}(t)$ is approximately constant, $\bar{E}(t) \approx \bar{E}\left(t_{2}\right)$.

The constant field $\bar{E}\left(t_{2}\right)$ acting on the interval $\left(t_{2}, t_{3}\right]$ excites new vacuum states which do not participate in the formation of the current density $\left\langle j^{1}(t)\right\rangle_{g}$ on the previous step. These new states have longitudinal momenta $p_{1} \operatorname{sgn}(E)$ greater than states excited from the vacuum during the time $t_{2}-t_{1}$ and transversal momenta $p_{2}$ limited by the field $\bar{E}\left(t_{2}\right)$. The region of these momenta is defined by inequalities similar to those in (64). Such inequalities in the mean field approximation have the form

$$
\begin{aligned}
& \left|p_{2}\right| \leq \sqrt{e\left|\bar{E}\left(t_{i}\right)\right| \hbar / v_{F}}\left[\sqrt{e\left|\bar{E}\left(t_{i}\right)\right| v_{F} / \hbar}\left(t-t_{i}\right)-K\right]^{1 / 2}, \\
& t_{i}+\frac{K}{\left|e \bar{E}\left(t_{i}\right) v_{F} / \hbar\right|^{1 / 2}} \leq-\frac{p_{1}}{e \bar{E}\left(t_{i}\right)} \leq t-\frac{K}{\left|e \bar{E}\left(t_{i}\right) v_{F} / \hbar\right|^{1 / 2}} .
\end{aligned}
$$

In the case $t \in\left(t_{2}, t_{3}\right]$, one has to set $t_{i}=t_{2}$ in these relations. As a result, the current density $\left\langle j^{1}(t)\right\rangle_{g}$ in the second interval takes the form

$$
\begin{aligned}
& \left\langle j^{1}(t)\right\rangle_{g}=\Delta j_{1}+\Delta j\left(t-t_{2}\right), \\
& \Delta j\left(t-t_{2}\right)=\operatorname{sgn}(E) D\left|\bar{E}\left(t_{2}\right)\right|^{3 / 2}\left(t-t_{2}\right),
\end{aligned}
$$

in the large $\tau$-limit. As in the case considered above, this current irradiates the corresponding field $E_{x}^{r a d}$, which is directed against to the constant field $\bar{E}\left(t_{2}\right)$, but its magnitude is less than the latter. Because $t-t_{2} \leq \Delta t_{2}$ and it is supposed that condition (87) holds true, one can see that the contribution from the derivative $\partial_{t} \bar{E}(t)$ to the current density $\left\langle j^{1}(t)\right\rangle_{g}$ is much less than the leading 
term, given by (91). Due to the contribution $E_{x}^{r a d}$, the mean field will decrease, $|\bar{E}(t)|<\left|\bar{E}\left(t_{2}\right)\right|$, reaching its minimal value at $t=t_{3}$. The further evolution of the vacuum mean current density and mean field has similar behavior for time $t$ from any interval $\Delta t_{i}$. In each such intervals the region of the momenta is given by Eq. (90). In the general case (in the corresponding large $\tau$-limit) when $t \in\left(t_{M}, t_{M+1}\right], 2 \leq M \leq N$, we can represent the current density $\left\langle j^{1}(t)\right\rangle_{g}$ as the following sum of partial contributions:

$$
\begin{aligned}
& \left\langle j^{1}(t)\right\rangle_{g}=\sum_{i=1}^{M-1} \Delta j_{i}+\Delta j\left(t-t_{M}\right), \\
& \Delta j_{i}=\operatorname{sgn}(E) D\left|\bar{E}\left(t_{i}\right)\right|^{3 / 2} \Delta t_{i}, \\
& \Delta j\left(t-t_{M}\right)=\operatorname{sgn}(E) D\left|\bar{E}\left(t_{M}\right)\right|^{3 / 2}\left(t-t_{M}\right) .
\end{aligned}
$$

Condition (87) guarantees that corrections to the leading terms $\Delta j_{i}$ and $\Delta j\left(t-t_{M}\right)$, given by (92), due to the derivatives $\partial_{t} \bar{E}(t)$ are small in each interval $\left(t_{i}, t_{i+1}\right]$. In each time interval, the mean current irradiates the corresponding field $E_{x}^{r a d}$ directed against the external field $E$, thus, the mean field $\bar{E}(t)$ is a monotonically decreasing function for $t \in\left(t_{i n}, t_{f i n}\right]$.

Let us use the approximations

$$
\begin{aligned}
& \left|\bar{E}\left(t_{i}\right)\right|^{3 / 2} \Delta t_{i} \approx \int_{t_{i}}^{t_{i+1}}|\bar{E}(t)|^{3 / 2} d t, \\
& \left|\bar{E}\left(t_{M}\right)\right|^{3 / 2}\left(t-t_{M}\right) \approx \int_{t_{M}}^{t}|\bar{E}(\tilde{t})|^{3 / 2} d \tilde{t} .
\end{aligned}
$$

Then the mean value $\left\langle j^{1}(t)\right\rangle_{g}$ can be approximated by the following integral form:

$$
\left\langle j^{1}(t)\right\rangle_{g}=\operatorname{sgn}(E) D \int_{t_{i n}}^{t}|\bar{E}(\tilde{t})|^{3 / 2} d \tilde{t} .
$$

Note that Eq. (93) holds true within large $\tau$-limit, in which we neglect a contribution from the derivative $\partial_{t} \bar{E}(t)$, if the condition (87) is valid for all the intervals $\left(t_{i}, t_{i+1}\right]$.

In a similar manner, one can find a unified representation of the vacuum mean current density $\left\langle j^{1}(t)\right\rangle_{g}$ and the diagonal elements $\left\langle T_{\mu \mu}(t)\right\rangle_{g}^{p}$ for the slowly varying electric field $\bar{E}(t)$ in the same approximation:

$$
\begin{aligned}
& \left\langle j^{1}(t)\right\rangle_{g}=\left.i 8 e \bar{P}_{1} \bar{\Delta}^{p}\left(x, x^{\prime}\right)\right|_{x=x^{\prime}}, \quad\left\langle T_{\mu \mu}(t)\right\rangle_{g}^{p}=\left.i 8 \bar{P}_{\mu}^{2} \bar{\Delta}^{p}\left(x, x^{\prime}\right)\right|_{x=x^{\prime}}, \\
& \bar{\Delta}^{p}\left(x, x^{\prime}\right)=-i \int_{t_{i n}+K \Delta t_{s t}}^{t-K \bar{h}_{\perp}} \bar{h}_{\perp}\left(\mathbf{x}_{\perp}, \mathbf{x}_{\perp}^{\prime}\right) \bar{h}_{\|}\left(x_{\|}, x_{\|}^{\prime}\right) d \tilde{t}, \\
& \bar{h}_{\|}\left(x_{\|}, x_{\|}^{\prime}\right)=\frac{|\bar{E}(\tilde{t})|}{\left|\bar{A}_{1}(t)\right|-\left|\bar{A}_{1}(\tilde{t})\right|} \exp \left[-\frac{i}{\hbar} e \bar{A}_{1}(\tilde{t})\left(x_{1}-x_{1}^{\prime}\right)\right] \cos \left\{\frac{1}{2}\left[\bar{\xi}\left(t^{\prime}\right)^{2}-\bar{\xi}(t)^{2}\right]\right\}, \\
& \bar{h}_{\perp}\left(x_{\perp}, x_{\perp}^{\prime}\right)=\left(\frac{v_{F}}{\hbar^{3}}\right)^{1 / 2} \frac{|e \bar{E}(\tilde{t})|^{1 / 2}}{(2 \pi)^{2}} \exp \left[-\frac{\left(x_{2}-x_{2}^{\prime}\right)^{2} e|\bar{E}(\tilde{t})|}{4 \pi \hbar v_{F}}\right]
\end{aligned}
$$

where

$$
\begin{aligned}
& \bar{P}_{0}=i \frac{\hbar}{v_{F}} \frac{\partial}{\partial t}, \quad \bar{P}_{1}=i \hbar \frac{\partial}{\partial x^{1}}+e \bar{A}_{1}(t), \quad \bar{P}_{2}=i \hbar \frac{\partial}{\partial x^{2}} \\
& \frac{\hbar}{v_{F}} \bar{\xi}(t) \frac{d \bar{\xi}(t)}{d t}=e\left[\left|\bar{A}_{1}(t)\right|-\left|\bar{A}_{1}(\tilde{t})\right|\right], \\
& \bar{A}_{1}(t)=\int_{t_{i n}}^{t} \bar{E}(\tilde{t}) d \tilde{t}+E t_{i n},
\end{aligned}
$$

$\overline{\Delta t}_{t}=\left(e|\bar{E}(t)| v_{F} / \hbar\right)^{-1 / 2}$, and $\Delta t_{s t}$ is the characteristic time-interval determined by Eq. (800). These equations are a generalization of representation (68) for the case under consideration, where $\bar{\Delta}^{p}\left(x, x^{\prime}\right)$ is a generalization of the function $\Delta^{p}\left(x, x^{\prime}\right)$ in (67). One can see that the expression $\left\langle j^{1}(t)\right\rangle_{g}$ given by (94) coincides with that given by Eq. (93) in the large $\tau$-limit. In this approximation the mean values $\left\langle T_{\mu \mu}(t)\right\rangle_{g}$ have the form:

$$
\begin{aligned}
& \left\langle T_{00}(t)\right\rangle_{g}=\left\langle T_{11}(t)\right\rangle_{g}=\left\langle T_{11}(t)\right\rangle_{g}^{p}, \\
& \left\langle T_{22}(t)\right\rangle_{g}=\left\langle T_{22}(t)\right\rangle_{g}^{p}+\left\langle T_{22}(t)\right\rangle_{g}^{c},
\end{aligned}
$$

with

$$
\begin{aligned}
\left\langle T_{11}(t)\right\rangle_{g}^{p}= & 2 e v_{F} \rho \int_{t_{i n}+K \Delta t_{s t}}^{t-K \overline{\Delta t}_{t}}|\bar{E}(\tilde{t})|^{3 / 2} \\
& \times\left[\left|\bar{A}_{1}(t)\right|-\left|\bar{A}_{1}(\tilde{t})\right|\right] d \tilde{t} \\
\left\langle T_{22}(t)\right\rangle_{g}^{p}= & \pi^{-1} \hbar \rho \int_{t_{i n}+K \Delta t_{s t}}^{t-K \overline{\Delta t}_{t}} \frac{|\bar{E}(\tilde{t})|^{5 / 2}}{\left|\bar{A}_{1}(t)\right|-\left|\bar{A}_{1}(\tilde{t})\right|} d \tilde{t} \\
\left\langle T_{22}(t)\right\rangle_{g}^{c}= & \frac{1}{2} \zeta_{R}(3 / 2) \hbar \rho|\bar{E}(t)|^{3 / 2}
\end{aligned}
$$

and $\rho=\pi^{-2} v_{F}^{-1 / 2} \hbar^{-3 / 2} e^{3 / 2}$.

By using Eq. (93), we obtain that in the Maxwell equations for the mean electromagnetic field the current term 
for $t \leq t_{\text {fin }}$ has the form $J=\left\langle j^{1}(t)\right\rangle_{g} \delta(z) \theta\left(t-t_{1}\right)$. The only nonzero components of the radiated field are the $x$ component $E_{x}^{r a d}$ of the electric field and the $y$-component $B_{y}^{r a d}$ of the magnetic field. Solving the Maxwell equations (81) under the initial conditions $E_{x}^{r a d}=0$ and $B_{y}^{r a d}=0$ at $t=t_{i n}$, one finds that for $0 \leq t-t_{i n} \leq$ $t_{\text {fin }}-t_{i n}$, and within a distance $|z|$ smaller than $c\left(t-t_{i n}\right)$ to the plane, the electromagnetic field produced by the current $\left\langle j^{1}(t)\right\rangle_{g}$ confined to the graphene sheet is:

$$
\begin{aligned}
E_{x}^{r a d}(t, z)=- & \operatorname{sgn}(E) \frac{\mu_{0} D}{2} \int|\bar{E}(t-l / c)|^{3 / 2} \\
& \times \theta\left(t-t_{i n}-l / c\right) \theta(l-|z|) d l, \\
B_{y}^{r a d}(t, z)= & \operatorname{sgn}(z) E_{x}^{r a d}(t, z) / c .
\end{aligned}
$$

It can be seen that the radiated field is a function of only the light-cone variable, $E_{x}^{r a d}(t, z)=E_{x}^{r a d}(t-|z| / c)$.

The electric field $E_{x}^{r a d}(t, z)$ has the same limit value near the plane from the upper and lower regions and we assume that $E_{x}^{r a d}(t, z)$ is continuous at $z=0$. Thus, inside the graphene film the irradiated electric field is $E_{x}^{r a d}(t)=E_{x}^{r a d}(t, 0)$. The mean magnetic field inside the graphene film is zero. Extracting the derivative $\partial_{t} \bar{E}(t)=$ $\partial_{t} E_{x}^{r a d}(t)$ from (97), we get that for $t \in\left(t_{i n}, t_{f i n}\right]$ a self-consistency equation for the mean field inside the graphene has the form:

$$
\frac{d|\bar{E}(t)|}{d t}=-\frac{4 \alpha}{\pi} \sqrt{\frac{e v_{F}}{\hbar}}|\bar{E}(t)|^{3 / 2}, \quad t \in\left(t_{i n}, t_{f i n}\right]
$$

As was already established, the mean field field $\bar{E}(t)$ is collinear to the external field $E$. Taking into account the initial condition, $\bar{E}\left(t_{i n}\right)=E$, one finds the solution of equation (98) as follows:

$$
\bar{E}(t)=E \epsilon(t)^{-2}, \quad \epsilon(t)=\left[1+\frac{2 \alpha}{\pi}\left(t-t_{i n}\right) / \Delta t_{s t}\right] .
$$

Then the irradiated field is $E_{x}^{r a d}(t)=\bar{E}(t)-E$.

As $t \rightarrow t_{f i n}$ the magnitude of the mean field $\bar{E}(t)$ decreases. Therefore, condition (88) will be satisfied for all the intervals $\left(t_{i}, t_{i+1}\right]$ if it is satisfied for the last interval $\left(t_{N}, t_{f i n}\right]$. In our approximation, this condition defines the minimal possible length of the intervals $\Delta t_{i}$,

$$
\Delta t_{i} \gg \overline{\Delta t}=\Delta t_{s t} \epsilon\left(t_{f i n}\right) .
$$

Condition (87) is satisfied for all the intervals $\left(t_{i}, t_{i+1}\right]$, if it is satisfied for the period $\left(t_{1}, t_{2}\right]$. With $\Delta t_{1} \gg \overline{\Delta t}$, the latter implies the following restriction for the interval $t_{\text {fin }}-t_{\text {in }}$ :

$$
\frac{4 \alpha}{\pi} \epsilon\left(t_{\text {fin }}\right) \ll 1 \Longrightarrow\left(t_{\text {fin }}-t_{\text {in }}\right) / \Delta t_{s t} \ll \frac{\pi^{2}}{8 \alpha^{2}} .
$$

The mean field approximation is consistent if inequality (101) holds true. This restriction is much weaker than the one given by Eq. (84), which ensures consistency of the external field approximation. Thus, in the approach under consideration the largest time scale is $\Delta t_{f i n}$, which is related to time scales considered before as follows:

$$
\Delta t_{f i n}=\frac{\pi}{2 \alpha} \Delta t_{b r}=\frac{\pi^{2}}{8 \alpha^{2}} \Delta t_{s t} .
$$

When $t_{f i n}-t_{\text {in }}$ approaches $\Delta t_{f i n}$, the magnitude $\left|\bar{E}\left(t_{\text {fin }}\right)\right|$ decreases and becomes of the order $\left|E_{\min }\right|=$ $|E|(4 \alpha / \pi)^{2}$. One can verify that in the case of ballistic transport, for all the typical experimental parameters, when the voltage varies from 1 to $5 \mathrm{~V}$ and sample lengths vary from 0.9 to $5.9 \mu \mathrm{m}$ (see [19]), the corresponding ballistic times satisfy condition (101), $T_{\text {bal }} \ll \Delta t_{\text {fin }}$. This means that in realistic cases the evolution of quantum states in course of the external field action satisfies the restrictions which justify the approximation under consideration, so that we can set $t_{\text {fin }}=t_{\text {out }}$.

Note that in the case when the field duration $t-t_{i n}$ exceeds $\Delta t_{\text {fin }}$ (that is unattainable for samples available at present), the mean field $\bar{E}(t)$ becomes too weak in comparison with the external field $E$, and then the model with pair creation by a constant external field fails to work. It is possible to consider the evolution of the mean field and the vacuum mean current for time intervals greater than $\Delta t_{\text {fin }}$ if one is able to calculate explicitly pair creation from vacuum due to a time-dependent electric field, which could be a task for further study. However, it is natural to assume that the residual effect of a weak electric field cannot significantly change the asymptotic behavior achieved by the time $t=t_{f i n}$, which effectively means that the effective external field duration is $T \approx t_{\text {fin }}-t_{\text {in }}$. That is why in what follows we set $t_{\text {fin }}=t_{\text {out }}$ for any $t_{\text {out }}$.

Substituting Eq. (99) in Eq. (93), we find the following result for the density of the vacuum mean current:

$$
\left\langle j^{1}(t)\right\rangle_{g}=\frac{e^{2} E}{2 \pi \hbar \alpha}\left[1-\epsilon(t)^{-2}\right], \quad t \in\left(t_{i n}, t_{f i n}\right] .
$$

Thus, we have found self-consistent solutions for mean field and vacuum mean current. From Eqs. (99) and (102) we see that at $\left(t-t_{1}\right) \gg \Delta t_{b r}=\Delta t_{s t} \pi / 4 \alpha$, the density of the vacuum mean current and radiated electric field take asymptotic forms:

$$
\left\langle j^{1}(t)\right\rangle_{g} \approx \frac{e^{2} E}{2 \pi \hbar \alpha}, \quad E_{x}^{r a d}(t) \approx-E .
$$

Thus, the self-consistent system of the mean field and the vacuum mean current at a given external electric field $E$ tends to a dynamic equilibrium state in which the external field inside the graphene is completely compensated by the radiated electric field. In this state the particle production is stopped and the vacuum mean current saturates. Close to this regime, the $I-V$ is almost linear. The momentum transferred by the applied force is limited by the finite value $e|E| \Delta t_{s t} \pi / 2 \alpha$. Due to the discrete structure of the lattice, formed by atoms at the carbon-carbon distance $a$, there is a natural momentum cutoff of the order $\hbar / a$. For this reason, instead of being 
restricted by the Bloch time $\Delta t_{B}$ mentioned in subsection IVA the applicability of the Dirac model is restricted, because of the crystalline structure of graphene, by the field strength $E_{B}$,

$$
E_{B} \sim\left(\frac{4 \alpha}{a}\right)^{2} \frac{\hbar v_{F}}{e} \sim 3 \times 10^{7} \mathrm{~V} / \mathrm{m}
$$

Substituting Eq. (99) into Eq. (96), we can explicitly find the diagonal elements $\left\langle T_{\mu \mu}(t)\right\rangle_{g}^{p}$. For example, the mean energy density reads:

$$
\left\langle T_{00}(t)\right\rangle_{g}=8 e v_{F} \rho|E|^{5 / 2}\left(\Delta t_{b r}\right)^{2}\left[\frac{1}{3}-\frac{1}{2 \epsilon(t)}+\frac{1}{6 \epsilon(t)^{3}}\right] .
$$

For $\left(t-t_{i n}\right) \gg \Delta t_{b r}$, we obtain from (104) the following asymptotic form:

$$
\left\langle T_{00}(t)\right\rangle_{g} \approx \frac{8}{3} e v_{F} \rho|E|^{5 / 2}\left(\Delta t_{b r}\right)^{2}
$$

One can see that $\left\langle T_{00}(t)\right\rangle_{g} \sim|E|^{3 / 2}$ asymptotically for large times, while for small intervals $\left(t-t_{i n}\right) \ll \Delta t_{b r}$ one has a dependence of the form $\left\langle T_{00}(t)\right\rangle_{g} \sim|E|^{5 / 2}$. The asymptotic behavior of the element $\left\langle T_{22}(t)\right\rangle_{g}^{p}$ reads:

$$
\left\langle T_{22}(t)\right\rangle_{g}^{p} \approx \frac{\hbar \rho}{3 \pi}|E|^{3 / 2}
$$

The behavior of the term $\left\langle T_{22}(t)\right\rangle_{g}^{c}$, given by (96), is completely determined by the field $|\bar{E}(t)|$ at a given time instant, which means that this term tends to zero asymptotically as $\epsilon(t)^{-3}$, and therefore can be neglected. We see that $\left\langle T_{00}(t)\right\rangle_{g}=\left\langle T_{11}(t)\right\rangle_{g} \gg\left\langle T_{22}(t)\right\rangle_{g}$ during the whole evolution up to asymptotically large values of the interval $\left(t-t_{i n}\right)$. Then, in this approximation, we have that trace $\left\langle T_{\mu}^{\mu}(t)\right\rangle_{g}=0$, as it can be expected for the equation of state for massless particles.

At $t=t_{\text {out }}$, the expressions (102) and (104) represent the current density and energy density of created particles, respectively, and the consistent mean electric field in graphene is given by Eq. (99). According to our problem setting, before the time moment $t_{\text {out }}$, we have an unitary evolution of a pure state of the Dirac-Maxwell system. At the initial time instant $t_{i n}$, this pure state is the vacuum for carriers and the coherent state of electromagnetic field with the initial mean value $E$. In the strong-field QED, "the measurement" which produces the decoherence (as a result of which we obtain many-particle state of carriers and a final state of the electromagnetic field specified by the mean value $\bar{E}\left(t_{\text {out }}\right)$ ) occurs not necessary at the moment when the external field switches off. This measurement can be done in any time instant after $t_{\text {out }}$ because the further evolution of the system is trivial and mean values remain unchanged. In the case of the finite graphene size and fixed constant voltage this is not true because in such a case we adapt our model with the $T$ constant external field to the situation where the effective duration is given, i.e., we set $T=T_{\text {eff }}$ and, therefore, by definition, we have $t_{\text {out }}=t_{\text {in }}+T$. (Note that one has to know the times $t_{i n}$ and $T$ in a different experimental situation for the measurement of the time evolution of the vacuum mean current, which has been been early proposed [4] but not implemented yet.) In this case, the time instant $t_{\text {out }}$ of switching off the effective electric field is understood as the effective time instant of the decoherence. We assume that the decoherence stops evolution of pure states in a sufficiently short time, so that the final mean electric current and EMT do not feel the effect of switching off the effective electric field.

When the effective duration $T_{\text {eff }}$ is due to the time scale of dissipation process $T_{d i s}=l_{m f p} / v_{F}$, we set $t_{\text {out }}-$ $t_{i n}=T_{\text {eff }}=l_{m f p} / v_{F}$, while in the ballistic case $l_{m f p}=$ $L_{x}$ and $T_{\text {eff }}=T_{b a l}$. Then, using (102), we obtain the $I-V$ curve in the following form:

$$
\begin{aligned}
& \left\langle j^{1}\left(t_{\text {out }}\right)\right\rangle_{g}=\frac{e^{2} V}{2 \pi \hbar \alpha L_{x}}\left[1-\epsilon\left(t_{\text {out }}\right)^{-2}\right], \\
& \epsilon\left(t_{\text {out }}\right)=1+\frac{2 \alpha T_{\text {eff }}}{\pi \Delta t_{\text {st }}}, \quad \frac{T_{\text {eff }}}{\Delta t_{\text {st }}}=\sqrt{\frac{e|V| l_{m f p}^{2}}{\hbar v_{F} L_{x}}} .
\end{aligned}
$$

Example 1: Considering a sample with $L_{x} \sim 1 \mu \mathrm{m}$ and a voltage $1 \mathrm{~V}$ in the ballistic case, we have $T_{b a l}=10^{-12} \mathrm{~s}$ and $|E|=10^{6} \mathrm{~V} / \mathrm{m}$. Alternatively, in the case of dissipation, we can consider a sample of arbitrary length, where the effective duration is $T_{\text {eff }}=10^{-12} \mathrm{~s}$, and the applied field strength is $|E|=|V| / L_{x}=10^{6} \mathrm{~V} / \mathrm{m}$. In both cases, $2 \alpha T_{\text {eff }}\left(\pi \Delta t_{s t}\right)^{-1}=0.18$, and condition (84) is satisfied. But then backreaction can be neglected and the $I-V$ curve from eq. (105) is close to $j \sim V^{3 / 2}$.

Example 2: Considering a sample with $L_{x} \sim 4 \mu \mathrm{m}$ and a voltage $4 \mathrm{~V}$ in the ballistic case, we have $2 \alpha T_{\text {eff }}\left(\pi \Delta t_{s t}\right)^{-1}=0.72$. Thus, condition (84) is not satisfied, and then the backreaction contribution in eq. (105) is important, resulting in an almost linear $I-V$. We see that our approach describe a transition from a superlinear to a linear $I-V$ curve when passing from low-mobility to high-mobility samples, in a manner similar to that observed in [19].

Note that outside the graphene film there is an irradiated electromagnetic plane wave $E_{x}^{r a d}(t, z)=E_{x}^{r a d}(t-$ $|z| / c$ ) of linear polarization given explicitly by Eqs. (97) and (99). Therefore, the energy dissipation due to this irradiation must be taken into account. In principle, such irradiation may be experimentally observed. It is the radiation due to the time-dependence of the mean current. We have calculated it nonperturbatively in the case when its electric component strength is comparable to the external field strength. Thus, the mechanism of the energy dissipation due to irradiation considered here differs essentially from that discussed in Ref. [18]. The authors of the latter work have treated the radiation due to the electron-hole recombination in the lowest order of the perturbation theory assuming large enough densities of carriers. That is why the relevance of such a consideration to the quantum transport close to the Dirac point is not clear. 


\section{SUMMARY}

In this summary, we briefly list the main new results obtained in the article and add some relevant comments. These results are collected in three blocks: I-General results in strong-field QED; II-Adaptation of the general results to the Dirac model in the graphene; III-Analysis of some immediate consequences to the graphene physics.

I. General results in strong-field QED

The one-loop renormalized mean current and EMT induced in the vacuum by the $T$-constant external electric field are computed in the framework of strong-field QED in spaces of arbitrary dimensions.

In the large $\tau$-limit, these quantities are represented as sums of local contributions due to the vacuum polarization, $\left(\left\langle j^{\mu}(t)\right\rangle_{\perp}^{c}\right.$ and $\left.\operatorname{Re}\left\langle T_{\mu \nu}(t)\right\rangle_{r e n}^{c}\right)$, and of global contributions due to the vacuum instability, $\left(\operatorname{Re}\left\langle j^{\mu}(t)\right\rangle^{p}\right.$ and $\left.\operatorname{Re}\left\langle T_{\mu \nu}(t)\right\rangle^{p}\right)$,

$$
\begin{aligned}
& \left\langle j^{\mu}(t)\right\rangle=\left\langle j^{\mu}(t)\right\rangle_{\perp}+\operatorname{Re}\left\langle j^{\mu}(t)\right\rangle_{\|}^{p}, \\
& \left\langle T_{\mu \nu}(t)\right\rangle_{\text {ren }}=\operatorname{Re}\left\langle T_{\mu \nu}(t)\right\rangle_{r e n}^{c}+\operatorname{Re}\left\langle T_{\mu \nu}(t)\right\rangle^{p} .
\end{aligned}
$$

These contributions are studied in detail. The vacuum polarization contributions to the EMT are expressed via the real part of the one-loop effective Euler-Heisenberg Lagrangian. In odd dimensions, unusual peculiarities of the vacuum polarization emerge: along with the longitudinal mean current, $\operatorname{Re}\left\langle j^{\mu}(t)\right\rangle_{\|}^{p}$ (which behaves in the same way as in even dimensions), there appears a transversal mean current, $\left\langle j^{\mu}(t)\right\rangle_{\perp}$. Its components correspond to fermions of different chiralities (pseudo-spin) moving in opposite directions. The sign of this current depends on the fermion species. That is an indication that the Chern-Simons term is present in a properly regularized effective action with odd number of fermion species. In the case of an external electric field, this transverse mean current depends essentially on the fermion mass (in contrast to the corresponding contributions to probability amplitudes of processes); in particular, it is zero in the massless case. Thus, the vacuum polarization in the electric field in odd dimensions is qualitatively different for mean values and for amplitudes of processes. This is the reason why the case of electric-like field differs substantially from the case of magnetic-like field. This fact is important in understanding properties of new materials where the electronic structure is described by the Dirac model of $(2+1)$-d fermions, in particular in the case of the interface transport in topological insulators, where there is only one fermion species. Moreover, it should be noted that even in the case of even number of fermion species with nonzero masses, neutral electron-hole pairs induced due to the applied electric field will be concentrated near lateral surfaces. Pairs with different chirality are concentrated on opposite lateral surfaces (there is a polarization with respect to the chirality). Otherwise, properties of these mean values in odd and even dimensions do not differ essentially. In particular, the longitudinal mean current components $\operatorname{Re}\left\langle j^{\mu}(t)\right\rangle_{\|}^{p}$ are lin- ear functions of $T$, and the energy density $\left\langle T_{00}(t)\right\rangle_{\text {ren }}$ and the pressure along the direction of the electric field $\left\langle T_{11}(t)\right\rangle_{\text {ren }}$ are quadratic functions of $T$.

II-Adaptation of the general results to the Dirac model in the graphene.

The general results described above are used in the non-perturbative consideration of electronic and energy quantum transport in graphene at low carrier density and low temperatures.

We have found: the time-dependence of the mean longitudinal current density $\left\langle j^{1}(t)\right\rangle_{g}$, the mean EMT $\left\langle T_{\mu \mu}(t)\right\rangle_{g}$, and the total number density of electron-hole pairs $n_{g}^{c r}$ created by the electric field in the graphene. In these calculations, we have used the strong-field approximation, the large $\tau$-limit (when the duration $T$ of the external electric field is sufficiently large), and the backreaction of created particles in screening the external field was taken into account. All these quantities are obtained for the case of an unitary evolution of a pure state of the Dirac-Maxwell system. To adopt our consideration to the existing experimental situations, where the length of graphene flakes is finite and a constant voltage is applied, the time $T$ was replaced by some effective time $T_{\text {eff }}$, which is determined by a certain decoherence. Assuming that this decoherence stops the evolution of pure states in sufficiently short times one can neglect effects of switching off the effective electric field on the final mean electric current and EMT.

The new approach proposed for treating the quantum transport in graphene allows to study unitary (coherent) evolution of system in the regime of large duration of a strong external field, when the electrodynamic backreaction is essential. In spite of the fact that we do not consider statistical effects of the decoherence, we believe that exact analysis of results of unitary evolution will be helpful as an initial stage for further considering the thermalization of the system.

It should be noted that in some high-energy physics problems (QED and QCD) in $(1+1)$ - and $(3+1)$ dimensions the backreaction from the pair production in electric fields was frequently discussed, in particular, in the framework of WKB approximation (see e.g. 37[39]) and using the numerical approach (see e.g. [40]). Recently, the exact solution for massless QED in 1+1dimensions was obtained, which by the definition takes the back reaction into account completely [41]. The authors of all these works considered only closed systems of fermions interacting with a mean electromagnetic field, in which the total energy was conserved. However, in considering the graphene in the framework of the Dirac model the backreaction due to the pair creation from the vacuum has never been studied. Our consideration of the backreaction in the graphene due to the pair creation is completely new and, moreover, is essentially different from that of the works cited above, because it concerns an open system with a source that maintains a given voltage.

III-Analysis of some immediate consequences to the 
graphene physics.

In the large $\tau$-limit, the mean values $\left\langle j^{1}(t)\right\rangle_{g}$ and $\left\langle T_{\mu \mu}(t)\right\rangle_{g}$ are proportional to the number density of pairs $r_{g}^{c r}$ of positive and negative charged states excited by the electric field per unit time. At the end of pure state evolution these quantities represent the current density and EMT of created particles, respectively, so that $\left\langle j^{1}(t)\right\rangle_{g} \sim|E|^{3 / 2}$ and $\left\langle T_{00}(t)\right\rangle_{g}=\left\langle T_{11}(t)\right\rangle_{g} \sim|E|^{5 / 2}$.

It is shown that there exists a parameter range $1 \ll$ $T / \Delta t_{s t} \ll \pi / 4 \alpha$ for which the external electric field of long duration $T$ is a good approximation of the effective mean field inside the graphene. Two corresponding characteristic time scales are established: one of them is $\Delta t_{s t}=\left(v_{F} e|E| / \hbar\right)^{-1 / 2}$, the time duration when the $\mathrm{dc}$ response goes from a linear to a non-linear regime and the effect of the real electric field is indistinguishable from the effect produced by a constant field; the other one is $\Delta t_{b r}=\Delta t_{s t} \pi / 4 \alpha$, the time duration when the backreaction becomes important. Such a specific regime is relevant to describe the experimentally observed superlinearity $j \sim V^{3 / 2}$ of the $I-V$ in low mobility samples.

A generalization of the Dirac model with the $T$ constant electric field is constructed that takes into account the backreaction of the mean current to the applied electric field set by a constant voltage. It is shown that in the case of graphene the electrodynamic backreaction is relevant and one can see the interplay between two and three dimensions: the time-dependent mean current is confined to the plane, but its radiation, forming the backreaction on the plane, escapes to the threedimensional space in the form of linearly polarized plane electromagnetic waves. A self-consistent solution of the Dirac-Maxwell set of equations for this generalized model is found and the effective mean field and effective mean values of the current and energy-momentum tensor are calculated. In this case the self-consistent system of the mean field and the vacuum mean current tends to a dynamic equilibrium state in which the external field inside the graphene is completely compensated by the radiated electric field, the particle production is stopped, and the vacuum mean current saturates. Close to this regime, the $I-V$ is almost linear and the mean energy density reads: $\left\langle T_{00}(t)\right\rangle_{g} \sim|E|^{3 / 2}$. This mean field approximation is consistent if the inequality $T / \Delta t_{s t} \ll \pi^{2} / 8 \alpha^{2}$ holds true. This restriction is much weaker than the one that determines consistency of the external-field approximation.

We show that non-linear and linear $I-V$ experimentally observed in low and high mobility samples, respectively, can be explained in the framework of the presented consideration and that such a behavior is a consequence of the fact that the conductivity in the graphene is essentially due to the pair creation from vacuum by the applied electric field.

\section{ACKNOWLEDGMENTS}

SPG acknowledges support of the program Bolsista CAPES/Brazil and thanks the University of São Paulo for hospitality. DMG acknowledges the permanent support of FAPESP and CNPq. SPG and DMG thank for partial support by the Grant No. 14.B37.21.0911 of Russian Ministry of Science. NY acknowledges support from CAPES (PRODOC program).
[1] D.M. Gitman, J. Phys. A 10, 2007 (1977); E.S. Fradkin and D.M. Gitman, Fortschr. Phys. 29, 381 (1981); E.S. Fradkin, D.M. Gitman and S.M. Shvartsman, Quantum Electrodynamics with Unstable Vacuum (Springer-Verlag, Berlin, 1991).

[2] W. Greiner, B. Müller and J. Rafelsky, Quantum electrodynamics of strong fields (Springer-Verlag, Berlin, 1985).

[3] R. Ruffini, G. Vereshchagin and S. Xue, Phys. Rep. 487, 1 (2010).

[4] D. Allor, T. D. Cohen and D. A. McGady, Phys. Rev. D 78, 096009 (2008).

[5] W. Heisenberg and H. Euler, Zeit. f. Phys. 98, 714 (1936); [English transl. arXiv:physics/0605038].

[6] G. V. Dunne, "Heisenberg-Euler effective lagrangians: basics and extensions", in Ian Kogan Memorial Volume, "From fields to strings: circumnavigating theoretical physics", ed. M. Shifman, A. Vainshtein and J. Wheater (World Scientific, 2005).

[7] A.I. Nikishov, Zh. Eksp. Teor. Fiz. 57, 1210 (1969) [Transl. Sov. Phys. JETP 30, 660 (1970)]; A. I. Nikishov, in "Quantum electrodynamics of phenomena in intense fields", Proc. P.N. Lebedev Phys. Inst. 111 (Nauka, Moscow,1979), p. 153.

[8] V.G. Bagrov, D.M. Gitman, and Sh.M. Shvartsman, Zh. Eksp. Teor. Fiz. 68, 392 (1975) [English transl. Sov.
Phys.-JETP, Vol. 41, 191 (1975)].

[9] S.P. Gavrilov, J. Phys. A: Math. Gen. 39, 6407 (2006).

[10] S. P. Gavrilov and D. M. Gitman, Phys. Rev. D 78, 045017 (2008).

[11] G. V. Dunne, Eur. Phys. J. D 55, 327 (2009).

[12] A. H. Castro Neto et al, Rev. Mod. Phys. 81, 109 (2009); N. M. R. Peres, Rev. Mod. Phys. 82, 2673 (2010); M.A.H. Vozmediano, M.I. Katsnelson, F. Guinea, Phys. Rep. 496, 109 (2010).

[13] D. Das Sarma, S. Adam, E. H. Hwang and E. Rossi, Rev. Mod. Phys. 83, 407 (2011).

[14] G. W. Semenoff, Phys. Rev. Lett. 53, 2449 (1984).

[15] B. Dóra and R. Moessner, Phys. Rev. B 81, 165431 (2010).

[16] M. Lewkowicz and B. Rosenstein, Phys. Rev. Lett. 102, 106802 (2009); B. Rosenstein, M. Lewkowicz, H. C. Kao and Y. Korniyenko, Phys. Rev. B 81, 041416(R) (2010).

[17] H. C. Kao, M. Lewkowicz and B. Rosenstein, Phys. Rev. B 82, 035406 (2010).

[18] M. Lewkowicz, H. C. Kao and B. Rosenstein, Phys. Rev. B 84, 035414 (2011).

[19] N. Vandecasteele, A. Barreiro, M. Lazzeri, A. Bachtold and F. Mauri, Phys. Rev. B 82, 045416 (2010).

[20] C.L. Fefferman and M.I. Weinstein, Honeycomb lattice potentials and Dirac points, arXiv:1202.3839 to appear 
in Journal of the American Mathematical Society.

[21] V.P. Gusynin, S.G. Sharapov, and J.P. Carbotte, Int. J. Mod. Phys. B. 21, 4611 (2007) ; O.V. Gamayun, E.V. Gorbar, and V.P. Gusynin, Phys. Rev. B 81, 075429 (2010); Y. Araki and G.W. Semenoff, Phys. Rev. B 86, 121402 (2012).

[22] M.Z. Hasan and C.L. Kane, Rev. Mod. Phys. 82, 3045 (2010); X.-L. Qi and S.-C. Zhang, Rev. Mod. Phys. 83, 1057 (2011).

[23] B. Büttner et al., Nature Physics, 7, 418 (2011).

[24] S. P. Gavrilov and D. M. Gitman, Phys. Rev. D 53, 7162 (1996).

[25] S.P. Gavrilov, D.M. Gitman, and J.L. Tomazelli, Nucl. Phys. B 795, 645 (2008).

[26] S. P. Gavrilov, D. M. Gitman, A. E. Goncalves, J. Math. Phys. 39, 3547 (1998).

[27] A. J. Niemi, G. W. Semenoff, Phys. Rev. Lett. 51, 2077 (1983).

[28] A. N. Redlich, Phys. Rev. Lett. 52, 18 (1984); Phys. Rev. D 29, 2366 (1984).

[29] B. Dóra and R. Moessner, Phys. Rev. B 83, 073403 (2011).
[30] J. Schwinger, Phys. Rev. 82, 749 (1951).

[31] E. Elizalde et al, "Zeta regularization techniques with applications" (World Scientific, Singapure, 1994).

[32] S. K. Blau, M. Visser and A. Wipf, Int. J. Mod. Phys. A 6, 5409 (1991).

[33] S. P. Gavrilov and D. M. Gitman, Phys. Rev. Lett. 101, 130403 (2008).

[34] P. R. Wallace, Phys. Rev. 71, 622 (1947).

[35] N. M. Vildanov, J. Phys.: Cond. Matt. 21, 445802 (2009).

[36] K. I. Bolotin et al, Phys. Rev. Lett. 101, 096802 (2008).

[37] I.D. Novikov, A.A. Starobinskii, Zh. Eksp. Teor. Fiz. 78, 3 (1980) [Engl. transl. Sov. Phys. JETP 51, 1 (1980)].

[38] Y. Kluger, E. Mottola, and J. M. Eisenberg, Phys. Rev. D 58, 125015 (1998).

[39] J.C.R Bloch, et al., Phys. Rev. D 60, 116011 (1999).

[40] J. F. Dawson, B. Mihaila and F. Cooper, Phys. Rev. D 80, 014011 (2009).

[41] Yi-Zen Chu and T. Vachaspati, Phys. Rev. D 81, 085020 (2010). 